This item was submitted to Loughborough's Research Repository by the author.

Items in Figshare are protected by copyright, with all rights reserved, unless otherwise indicated.

\title{
Composite adaptive control of a robotic joint for passive deployment
} applications

PLEASE CITE THE PUBLISHED VERSION

PUBLISHER

Professional Engineering Publishing / @ IMECHE

VERSION

VoR (Version of Record)

LICENCE

CC BY-NC-ND 4.0

\section{REPOSITORY RECORD}

Reedman, Adam V.C., and Kaddour Bouazza-Marouf. 2019. "Composite Adaptive Control of a Robotic Joint for Passive Deployment Applications". figshare. https://hdl.handle.net/2134/5147. 
This item was submitted to Loughborough's Institutional Repository (https://dspace.lboro.ac.uk/) by the author and is made available under the following Creative Commons Licence conditions.

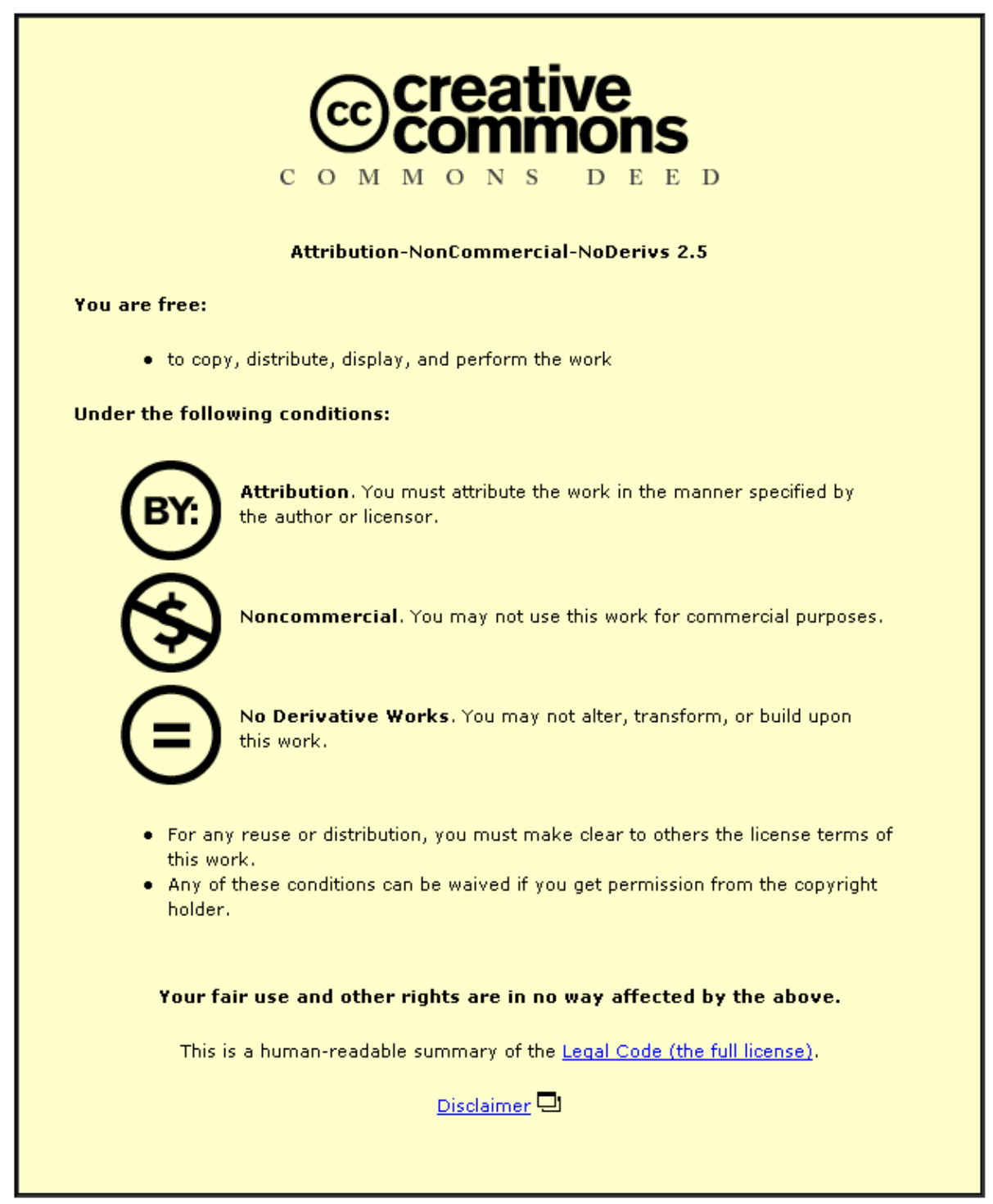

For the full text of this licence, please go to: http://creativecommons.org/licenses/by-nc-nd/2.5/ 


\title{
Composite adaptive control of a robotic joint for passive deployment applications
}

A V C Reedman and K Bouazza-Marouf*

Wolfson School of Mechanical and Manufacturing Engineering, Loughborough University, Leicestershire, UK

\begin{abstract}
A composite adaptive control scheme for the control of an actively constrained revolute joint with backlash cancellation is presented in this paper. The drive mechanism consists of two motor-driven worms coupled to a single worm wheel. The mathematical model and control strategies are reviewed. This is followed by the derivation of the composite adaptive controllers. Simulation and experimental results show that the composite adaptive control scheme gives an equivalent performance to a computed-torque algorithm without compromising the mechanism's ability to cancel backlash.
\end{abstract}

Keywords: backlash cancellation, composite adaptive control, robotics

\begin{tabular}{|c|c|c|c|}
\hline \multicolumn{2}{|c|}{ NOTATION } & \multirow{4}{*}{$\begin{array}{l}\mathbf{P}_{\alpha}, \mathbf{P}_{\beta} \\
r \\
r_{\alpha}\end{array}$} & \multirow{2}{*}{$\begin{array}{l}\text { adaptive update law gain matrix for } \alpha \\
\text { and } \beta \text { unwinding } \\
\text { filtered tracking error }\end{array}$} \\
\hline$C_{\mathrm{fa}}$ & viscous friction coefficient of the $\alpha$ worm & & \\
\hline$\sigma_{\mathrm{f} \alpha}$ & system & & radius of contact of the $\alpha$ worm with the \\
\hline$C_{\mathrm{f} \beta}$ & viscous friction coefficient of the $\beta$ worm & & worm-wheel \\
\hline$C_{\mathrm{f} \theta}$ & $\begin{array}{l}\text { system } \\
\text { viscous friction coefficient of the }\end{array}$ & $r_{\beta}$ & $\begin{array}{l}\text { radius of contact of the } \beta \text { worm with the } \\
\text { worm-wheel }\end{array}$ \\
\hline$C_{\mathrm{T} \alpha}, C_{\mathrm{T} \beta}$ & $\begin{array}{l}\text { worm-wheel system } \\
\text { total system viscous friction with respect }\end{array}$ & $r_{\theta}$ & $\begin{array}{l}\text { radius of contact of the worm-wheel with } \\
\alpha \text { and } \beta \text { worms }\end{array}$ \\
\hline$C_{\alpha}$ & $\begin{array}{l}\text { to the } \alpha \text { and } \beta \text { motor torque } \\
\text { equivalent viscous friction coefficient of } \\
\text { the } \alpha \text { worm }\end{array}$ & $\mathbf{W}_{\alpha}, \mathbf{W}_{\beta}$ & $\begin{array}{l}\text { regression matrix for } \alpha \text { and } \beta \text { unwinding } \\
\text { control of the rotational system } \\
\text { filtered regression matrix }\end{array}$ \\
\hline$C_{\beta}$ & $\begin{array}{l}\text { equivalent viscous friction coefficient of } \\
\text { the } \beta \text { worm system }\end{array}$ & $x^{\mathrm{f}_{\mathrm{f}}, \mathrm{w}_{\mathrm{f}} \beta}$ & $\begin{array}{l}\text { equivalent linear displacement of the } \\
\alpha \text { worm }\end{array}$ \\
\hline$C_{\theta}$ & $\begin{array}{l}\text { equivalent viscous friction coefficient of } \\
\text { the worm-wheel system }\end{array}$ & $y$ & $\begin{array}{l}\text { equivalent linear displacement of the } \\
\beta \text { worm }\end{array}$ \\
\hline $\begin{array}{l}e \\
J_{\mathrm{T} \alpha}, J_{\mathrm{T} \beta}\end{array}$ & $\begin{array}{l}\text { joint tracking error } \\
\text { total system inertia with respect to the } \alpha \\
\text { and } \beta \text { motor torque }\end{array}$ & $\mathbf{Y}_{\alpha}, \mathbf{Y}_{\beta}$ & $\begin{array}{l}\text { regression matrix for } \alpha \text { and } \beta \text { unwinding } \\
\text { control of the filtered tracking error } \\
\text { system }\end{array}$ \\
\hline $\begin{array}{l}J_{\alpha} \\
J_{\beta}\end{array}$ & $\begin{array}{l}\text { inertia of the } \alpha \text { worm and motor } \\
\text { inertia of the } \beta \text { worm and motor }\end{array}$ & $z$ & $\begin{array}{l}\text { equivalent linear displacement of the } \\
\text { worm-wheel }\end{array}$ \\
\hline$J_{\theta}^{\prime}$ & $\begin{array}{l}\text { inertia of the worm-wheel, shaft and } \\
\text { robot arm }\end{array}$ & $\gamma$ & lead angle of $\alpha$ and $\beta$ worms \\
\hline$K_{\mathrm{va}}, K_{\mathrm{v} \beta}$ & controller gains for $\alpha$ and $\beta$ unwinding & $\begin{array}{l}\varepsilon \\
\theta\end{array}$ & friction constant \\
\hline & $\begin{array}{l}\text { equivalent mass of } J_{\alpha} \\
\text { equivalent mass of } J_{Q}\end{array}$ & $\theta_{\mathrm{d}}$ & desired link position \\
\hline & equivalent mass of $J_{\theta}^{\beta}$ & $\mu_{\mathrm{d} \alpha}, \mu_{\mathrm{d} \beta}$ & $\begin{array}{l}\text { coefficient of dynamic friction of } \alpha \text { and } \\
\beta \text { worms }\end{array}$ \\
\hline $\begin{array}{l}\text { The MS w } \\
\text { revision for } \\
\text { * Correspor } \\
\text { Manufactur } \\
\text { Leicestersh }\end{array}$ & $\begin{array}{l}\text { ceived on } 14 \text { September } 2001 \text { and was accepted after } \\
\text { ication on } 26 \text { February } 2002 . \\
\text { author: Wolfson School of Mechanical and } \\
\text { Egineering, Loughborough University, Loughborough, } \\
\text { E11 3TU, UK. }\end{array}$ & $\begin{array}{l}\mu_{\mathrm{s} \alpha}, \mu_{\mathrm{s} \beta} \\
\tau_{\mathrm{f} \alpha}, \tau_{\mathrm{f} \beta} \\
\tau_{\mathrm{m} \alpha}, \tau_{\mathrm{m} \beta}\end{array}$ & $\begin{array}{l}\text { coefficient of static friction of } \alpha \text { and } \\
\beta \text { worms } \\
\text { filtered } \alpha \text { and } \beta \text { motor torque } \\
\text { torque generated by driving } \alpha \text { and } \\
\beta \text { motors }\end{array}$ \\
\hline
\end{tabular}


$\tau_{\mathrm{u}}$ equivalent torque generated by applied user-input force

$\hat{\boldsymbol{\Phi}}_{\alpha}, \hat{\boldsymbol{\Phi}}_{\beta}^{\beta}$ vector of system parameters vector of system parameter estimates

\section{INTRODUCTION}

Demand for robotic devices that interact closely with humans is increasing. For surgical intervention, robotic devices have been introduced into the operating theatre to help the surgeon perform surgical procedures with a higher degree of accuracy and reliability. However, many of the robotic devices currently in use are based on modified industrial manipulators and have typically been designed for high-speed, high-torque applications. The introduction of a large, powerful robot into an environment such as the operating theatre casts doubt on the safety of the patient, surgeon and other operating room staff $[1]$.

To this end, research in the field of robot-assisted surgery has increasingly been directed at making the surgeon physically interact with custom-built robotic devices. By grasping a control handle the user is required to apply a force to the manipulator in order to make it move. These devices have been classified as either passive or active. A passive device is one that cannot provide a force/torque to the links of the robot that would cause motion. The classic example of a passive device is PADyC [2]. At every joint PADyC has a pair of overrunning clutches, each running on a separate motordriven hub. By controlling the speed of each motor one clutch limits the maximum allowable speed in the clockwise direction while the second clutch restricts speed in the anticlockwise direction. It was demonstrated that PADyC could be used to confine the motion of the surgeon within a predefined work area and, to a limited extent, follow a predefined path. With $20 \mathrm{~N}$ of force applied on the control handle, the two-degree-offreedom (DOF) prototype of PADyC exhibited up to $20 \mathrm{~mm}$ of error at the tool tip with link lengths of $0.25 \mathrm{~m}$. The error was attributed to joint flexibility and backlash in the clutch mechanism. Errors of this magnitude cannot be tolerated in most surgical applications.

Another passive device, the 3-DOF COBOT (collaborative robot) developed at Northwestern University (Illinois), uses a continuously variable transmission (CVT) for each joint [3]. Each CVT consists of a sphere, a pair of separate drive rollers coupled to output shafts and two steering wheels that control the relative velocities of the drive shafts. By coupling these CVTs to a common drive unit the robot is kinematically constrained to follow the desired path, i.e. forcing motion of one joint causes motion of the end-effector along the path. However, as the CVT relies on a limited amount of friction between the drive rollers and the sphere to maintain position, the user can overpower the CVT, forcing it to deviate from the path.

Active devices, on the other hand, use motors directly coupled to the joints to provide a motive force. ACROBOT, developed at Imperial College (London), is a 4-DOF manipulator designed to help the surgeon perform total knee replacement surgery [4]. The surgeon controls the motion of the manipulator by applying a force to the control handle that is attached to the endeffector. The motorized joints are controlled to allow motion in the direction required by the surgeon. All of the joints are backdriveable and a d.c. motor at each joint controls the resistive force that the surgeon feels using a force control strategy. The workspace of the robot is actively constrained to confine the end-effector within a pre-planned safe working region. However, while the ACROBOT system requires physical input from the surgeon in order to move the manipulator, the force-controlled servomotors in each joint are still powerful enough to provide motion against the user. This again raises many issues regarding surgeon and patient safety. Although passive devices potentially have significant advantages over active mechanisms in terms of safety, thus far the positional accuracy of such devices while tracking a predefined path/trajectory has been absent.

In a previous paper, an actively constrained, revolute robotic joint was presented for use in applications where direct interaction with humans is required [5]. The nonbackdriveable dual-worm mechanism was shown to be capable of cancelling the effects of backlash. A mathematical model of the mechanism was presented and was used to develop a computed-torque control algorithm for tracking a desired path. The algorithm guarantees exact tracking when the robot parameters are known exactly. However, non-linear robot parameters (including frictional and inertial terms) are notoriously difficult to estimate. In this paper, a composite adaptive control scheme is presented that is used to estimate system parameters such as inertia, damping coefficients and friction coefficients. A review of the concept of the dual-worm robot joint mechanism is given, followed by a statement of the mathematical model of the gear system. The strategy for control of the mechanism is presented along with a discussion on the selection of an appropriate adaptive control scheme. Simulation and experimental results are presented showing a comparison between the performance of the computed torque and composite adaptive controllers.

\section{CONCEPTUAL OVERVIEW}

In gear system design, a small amount of backlash is required in order to allow for thermal expansion, lubrication and lower frictional losses within a drive train [6]. In many robotic applications, even minute amounts 


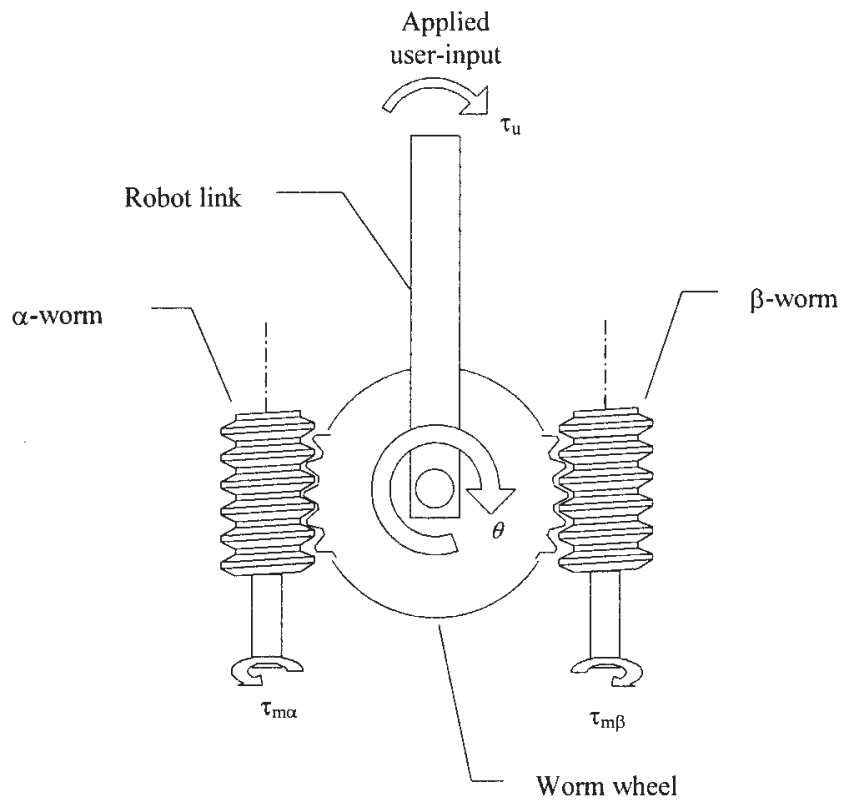

Fig. 1 Dual-worm drive joint

of backlash can cause errors in position that are not acceptable. For example, if a single link of a robot arm exhibits $\pm 0.0087 \mathrm{rad}$ ( or $\pm 0.5^{\circ}$ ) of backlash there will be an error of $\pm 3.5 \mathrm{~mm}$ at the end of a $0.4 \mathrm{~m}$ link.

The proposed system is a robotic joint designed to cancel backlash. The joint, shown schematically in Fig. 1, consists of two worms each driven by a low-power d.c. servomotor. The two worms follow a worm-wheel that is fixed to the robot link. The user provides a force on the end-effector and motion is allowed by controlling the two worms simultaneously. The nonbackdriveability of the worm mechanism means that no matter how much force the operator applies, motion is not allowed until both motors are controlled to move in the same direction.

The parallel nature of this overactuated joint structure makes this active system safer than modified industrial robots. If there was a failure, both motors would have to drive in the same direction for any motion to occur. However, if this failure occurs, acceleration of the joints would be very small, due to the inertia of the links and low power of the motors, giving the operator plenty of time to react, i.e. to release the dead-man's handle. The dual-worm mechanism also has the ability to eliminate backlash using a control strategy which is discussed below. The proposed control of the joint is robust against the non-linear effects of inertia and frictional forces at the worm/wheel interface.

\section{MATHEMATICAL MODEL}

The mathematical model of the dual-worm driven mechanism, developed in Reedman and BouazzaMarouf [5], is given by

$$
J_{\mathrm{T}} \theta+C_{\mathrm{T}} \theta=\tau_{\mathrm{u}}+\frac{\tau_{\mathrm{m} \alpha} r_{\theta}}{\Delta_{\alpha} r_{\alpha}}+\frac{\tau_{\mathrm{m} \beta} r_{\theta}}{\Delta_{\beta} r_{\beta}}
$$

where

$$
\begin{aligned}
& J_{\mathrm{T}}=\left(J_{\theta}+\frac{J_{\alpha} r_{\theta}^{2}}{\Delta_{\alpha} r_{\alpha}^{2} \tan \gamma}+\frac{J_{\beta} r_{\theta}^{2}}{\Delta_{\beta} r_{\beta}^{2} \tan \gamma}\right) \\
& C_{\mathrm{T}}=\left(C_{\mathrm{f} \theta}+\frac{C_{\mathrm{f} \alpha} r_{\theta}^{2}}{\Delta_{\mathrm{a}} r_{\alpha}^{2} \tan \gamma}+\frac{C_{\mathrm{f} \beta} r_{\theta}^{2}}{\Delta_{\beta} r^{2} \tan \gamma}\right) \\
& \Delta_{\alpha}=\frac{\sin \gamma+\left[\mu_{\mathrm{d} \beta}+\left(\mu_{\mathrm{s} \beta}-\mu_{\mathrm{d} \beta}\right) \mathrm{e}^{\left.-\left.\right|^{\theta \mid} / \varepsilon \sin \gamma\right)}\right] \operatorname{sgn}(\theta) \cos \gamma}{\cos \gamma-\left[\mu_{\mathrm{d} \beta}+\left(\mu_{\mathrm{s} \beta}-\mu_{\mathrm{d} \beta}\right) \mathrm{e}^{\left.-\left.\right|^{\theta \mid / \varepsilon} \sin \gamma\right)}\right] \operatorname{sgn}(\theta) \sin \gamma} \\
& \Delta_{\beta}=\frac{\sin \gamma-\left[\mu_{\mathrm{d} \beta}+\left(\mu_{\mathrm{s} \beta}-\mu_{\mathrm{d} \beta}\right) \mathrm{e}^{\left.-\left.\right|^{\theta \mid / \varepsilon} \sin \gamma\right)}\right] \operatorname{sgn}(\theta) \cos \gamma}{\cos \gamma+\left[\mu_{\mathrm{d} \beta}+\left(\mu_{\mathrm{s} \beta}-\mu_{\mathrm{d} \beta}\right) \mathrm{e}^{\left.-\left.\right|^{\theta \mid /(\varepsilon} \sin \gamma\right)}\right] \operatorname{sgn}(\theta) \sin \gamma}
\end{aligned}
$$

The friction forces between the two worms and the wheel are modelled using an exponential stick-slip friction model [7].

\section{CONTROL STRATEGY}

The aim of the control algorithm is to make the manipulator track a desired position command while cancelling backlash at the worm interface. In order to ensure surgeon and patient safety, the joint and control method must not exhibit:

(a) any motion against the user,

(b) any motion without direct control from the user or

(c) any backlash at the worm/wheel interface.

To this end, effective control of the dual-worm mechanism requires two algorithms. In the clockwise direction (i.e. when the equivalent torque generated by the userinput force, $\tau_{\mathrm{u}}>0$ ) the motor command voltage for the $\alpha$ worm is set to a constant value and the $\beta$ motor torque, $\tau_{\mathrm{m} \beta}$, is controlled to unwind the $\beta$ worm to track the trajectory. In this condition the $\beta$ worm leads and the $\alpha$ worm is used to follow the motion of the worm-wheel without applying unnecessary frictional forces to the system. Control of motion in this manner will be termed $\beta$ unwinding control. However, in the anticlockwise direction (i.e. $\tau_{\mathrm{u}}<0$ ) the $\beta$ worm motor command voltage is set to a constant value and $\tau_{\mathrm{m} \alpha}$ is used to control the unwinding of the $\alpha$ worm to track the trajectory. This is termed $\alpha$ unwinding control. In this instance, the $\alpha$ worm leads and the $\beta$ worm follows. This dual-control strategy with the proposed control algorithm is shown to work well.

The velocity command is generated from the userinput force in the following way: 


$$
\theta_{\mathrm{d}}= \begin{cases}0 & \forall\left|\tau_{\mathrm{u}}\right|<\tau_{\mathrm{u}, \min } \\ \theta_{\mathrm{d}, \max } \operatorname{sgn}\left(\tau_{\mathrm{u}}\right)\left[-\frac{2\left|\tau_{\mathrm{u}}-\tau_{\mathrm{u}, \min }\right|^{3}}{\left(\tau_{\mathrm{u}, \max }-\tau_{\mathrm{u}, \min }\right)^{3}}+\frac{3\left|\tau_{\mathrm{u}}-\tau_{\mathrm{u}, \min }\right|^{2}}{\left(\tau_{\mathrm{u}, \max }-\tau_{\mathrm{u}, \min }\right)^{2}}\right] & \forall \tau_{\mathrm{u}, \min } \leqslant\left|\tau_{\mathrm{u}}\right| \leqslant \tau_{\mathrm{u}, \max } \\ \theta_{\mathrm{d}, \max } \operatorname{sgn}\left(\tau_{\mathrm{u}}\right) & \forall\left|\tau_{\mathrm{u}}\right|>\tau_{\mathrm{u}, \max }\end{cases}
$$

where $\theta_{\mathrm{d}, \max }, \tau_{\mathrm{u}, \min }$ and $\tau_{\mathrm{u}, \max }$ are positive constants chosen to give a smooth motion from the mechanism. The velocity-limiting algorithm of equation (2), shown graphically in Fig. 2, is different from that used in Reedman and Bouazza-Marouf [5]. This strategy has been employed to ensure sufficient smoothness of the desired position, velocity and acceleration command signals.

Craig et al. [8] presented an adaptive control scheme for the control of robotic systems that ensures convergence of the parameter error to zero under certain conditions on the desired trajectory, known as persistency of excitation. The control method that has been proposed here involves switching between two controllers: one controller for controlling motion in the positive direction and another for the negative direction. Subsequently, it is not possible to generate a persistently exciting trajectory for each controller. The control method of Craig et al. also requires inversion of the manipulator inertia matrix and measurement of acceleration. Although the parameter resetting technique used in reference $[\mathbf{8}$ ] ensures the existence of the inverse of the inertia matrix, the poor acceleration measurement (derived by twice differentiating the position with respect to time) is also undesirable.

Due to the requirement of acceleration measurement and the inability to generate a persistently exciting trajectory of the proposed control strategy, a control method that relies on a less restrictive condition than persistency of excitation, known as the infinite integral condition, is used. Slotine and Li [9] proposed a composite adaptive controller that extracts information from both the tracking error and a prediction error in the filtered joint torque. This method consists of filtering the joint torque, making an estimate of this filtered quantity, designing a controller to track the desired trajectory and finally se-

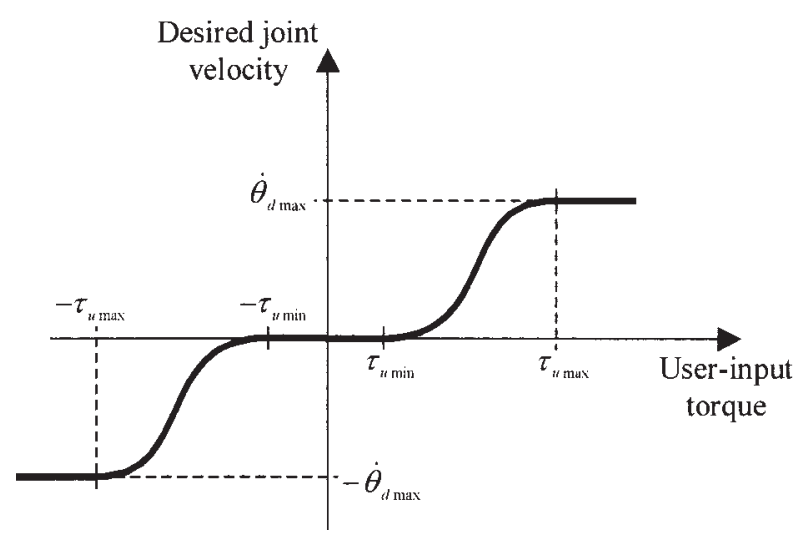

Fig. 2 User defined velocity demand lecting a parameter update rule that ensures convergence of both the tracking and parameter errors to zero. This torque filtering method also removes the need for acceleration measurements and inversion of the manipulator inertia matrix.

\section{COMPOSITE ADAPTIVE CONTROLLER}

In the development of the composite adaptive controller, the error between the desired position and the actual position of the link is defined by

$$
e=\theta_{\mathrm{d}}-\theta
$$

and the quantity $r$, known as the filtered tracking error, is defined by

$$
r=\dot{e}+\lambda e
$$

Both $\alpha$ unwinding and $\beta$ unwinding control strategies are developed below, followed by a stability analysis of the controllers.

\section{$5.1 \alpha$ unwinding control}

The equation of motion for the system of gears, given in equation (1), can be used to solve for the $\alpha$ motor torque, $\tau_{\mathrm{m} \alpha}$, by multiplying both sides by $\Delta_{\alpha} r_{\alpha} / r_{\theta}$; i.e.

$$
\tau_{\mathrm{m} \alpha}=J_{\mathrm{T} \alpha} \theta+C_{\mathrm{T} \alpha} \theta-\tau_{\mathrm{u}} \frac{\Delta_{\alpha} r_{\alpha}}{r_{\theta}}-\tau_{\mathrm{m}} \beta \frac{\Delta_{\alpha} r_{\alpha}}{\Delta_{\beta} r_{\beta}}
$$

where

$$
J_{\mathrm{T} \alpha}=J_{\mathrm{T}} \frac{\Delta_{\alpha} r_{\alpha}}{r_{\theta}}, \quad C_{\mathrm{T} \alpha}=C_{\mathrm{T}} \frac{\Delta_{\alpha} r_{\alpha}}{r_{\theta}}
$$

The assumption is made that the transition between static and dynamic friction is very fast (because of the $1 / \sin \gamma$ term in both $\Delta_{\alpha}$ and $\Delta_{\beta}$ ); hence, once motion has started $\Delta_{\alpha}$ and $\Delta_{\beta}$ are constant. Under this assumption, it is possible to write $\tau_{\mathrm{m} \alpha}$ as a linear combination of constant unknown parameters, $\boldsymbol{\Phi}_{\alpha}$, and known functions, $\mathbf{W}_{\alpha}$. Therefore, equation (5) can be written as

$$
\tau_{\mathrm{m} \alpha}=\mathbf{W}_{\alpha} \boldsymbol{\Phi}_{\alpha}
$$

where

$$
\begin{aligned}
& \mathbf{W}_{\alpha}=\left[\begin{array}{llll}
\theta & \theta & \tau_{\mathrm{u}} & \tau_{\mathrm{m} \beta}
\end{array}\right] \\
& \boldsymbol{\Phi}_{\alpha}=\left[\begin{array}{llll}
J_{\mathrm{T} \alpha} & C_{\mathrm{T} \alpha} & -\frac{\Delta_{\alpha} r_{\alpha}}{r_{\theta}} & -\frac{\Delta_{\alpha} r_{\alpha}}{\Delta_{\beta} r_{\beta}}
\end{array}\right]^{\mathrm{T}}
\end{aligned}
$$

I06701 C IMechE 2002 
Equation (6) can also be written as

$$
\tau_{\mathrm{m} \alpha}=\dot{h}_{\alpha}+g_{\alpha}
$$

where

$$
\dot{h}_{\alpha}=\frac{\partial}{\partial t}\left(J_{\mathrm{T} \alpha} \theta\right)
$$

and

$$
g_{\alpha}=-\dot{J}_{\mathrm{T} \alpha} \theta+C_{\mathrm{T} \alpha} \theta-\tau_{\mathrm{u}} \frac{\Delta_{\alpha} r_{\alpha}}{r_{\theta}}-\tau_{\mathrm{m} \beta} \frac{\Delta_{\alpha} r_{\alpha}}{\Delta_{\beta} r_{\beta}}
$$

Equation (5) has now been separated in a way that allows the acceleration term, $\theta$, to be filtered out. By filtering both sides of equation (7) it is possible to write the filtered torque equation given by

$$
\tau_{\mathrm{f} \alpha}=f^{*} \tau_{\mathrm{m} \alpha}=f^{*} \hat{h}_{\alpha}+f^{*} g_{\alpha}
$$

where $f$ is the impulse response of the linear stable, strictly proper filter, and * is used to denote the convolution operation. By the property of convolution, it is also possible to write

$$
f^{*} \dot{h}_{\alpha}=\int_{0}^{t} f(t-v) \dot{h}_{\alpha}(v) \mathrm{d} v
$$

and integrating by parts yields

$$
f^{*} \dot{h}_{\alpha}=-\dot{f}^{*} h_{\alpha}+f(0) h_{\alpha}-f h_{\alpha}(0)
$$

Substitution of equation (10) into equation (8) and noting that $J_{\mathrm{T} \alpha}$ is constant and the filter and velocity are initialized to $f(0)=c$ and $\theta(0)=0$ respectively yields

$$
\begin{aligned}
\tau_{\mathrm{f} \alpha}= & -f^{*}\left(J_{\mathrm{T} \alpha} \theta\right)+c J_{\mathrm{T} \alpha} \theta \\
& +f^{*}\left(C_{\mathrm{T} \alpha} \theta-\tau_{\mathrm{u}} \frac{\Delta_{\alpha} r_{\alpha}}{r_{\theta}}-\tau_{\mathrm{m} \beta} \frac{\Delta_{\alpha} r_{\alpha}}{\Delta_{\beta} r_{\beta}}\right) \\
= & \mathbf{W}_{\mathrm{f} \alpha} \boldsymbol{\Phi}_{\alpha}
\end{aligned}
$$

where

$$
\mathbf{W}_{\mathrm{f} \alpha}=\left[\begin{array}{llll}
-f * \theta+c \theta & f * \theta & f^{*} \tau_{\mathrm{u}} & f^{*} \tau_{\mathrm{m} \beta}
\end{array}\right]
$$

$\mathbf{W}_{\mathrm{fa}}$ is referred to as the filtered regression matrix and $\boldsymbol{\Phi}_{\alpha}$ is the same as in equation (6). Assuming that $\tau_{\mathrm{m} \alpha}$, $\tau_{\mathrm{m} \beta}, \tau_{\mathrm{u}}$ and $\theta$ are measurable, i.e. that the filtered regression matrix is known, it is possible to define the estimate of the filtered $\alpha$ motor torque, $\hat{\tau}_{\mathrm{f} \alpha}$, based on the estimate of the unknown parameters, $\boldsymbol{\Phi}_{\alpha}$, such that

$$
\hat{\tau}_{\mathrm{f} \alpha}=\mathbf{W}_{\mathrm{f} \alpha} \hat{\boldsymbol{\Phi}}_{\alpha}
$$

The error in the estimate of the filtered torque is defined as

$$
\tau_{\mathrm{f} \alpha}=\tau_{\mathrm{f} \alpha}-\tau_{\mathrm{f} \alpha}
$$

In order to design a controller for the system, consider the Lyapunov-like function given by

$$
V_{\alpha}=\frac{1}{2} J_{\mathrm{T} \alpha} r^{2}+\frac{1}{2} \boldsymbol{\Phi}_{\alpha}^{\mathrm{T}} \mathbf{P}_{\alpha}^{-1} \boldsymbol{\Phi}_{\alpha}
$$

and its time derivative

$$
\dot{V}_{\alpha}=J_{\mathrm{T} \alpha} \dot{r} r+\boldsymbol{\Phi}_{\alpha}^{\mathrm{T}} \mathbf{P}_{\alpha}^{-1} \dot{\boldsymbol{\Phi}}_{\alpha}+\frac{1}{2} \boldsymbol{\Phi}_{\alpha}^{\mathrm{T}} \dot{\mathbf{P}}_{\alpha}^{-1} \boldsymbol{\Phi}_{\alpha}
$$

where $r=e+\lambda e$ is the filtered tracking error, $e=\theta_{\mathrm{d}}-\theta$ is the tracking error, $\boldsymbol{\Phi}_{\alpha}=\boldsymbol{\Phi}_{\alpha}-\boldsymbol{\Phi}_{\alpha}$ is the error in the parameter estimates and $\mathbf{P}_{\alpha}$ is the time-varying symmetric matrix. Making the following observation:

$$
J_{\mathrm{T} \alpha} r^{r}=J_{\mathrm{T} \alpha}\left(\theta_{\mathrm{d}}+\lambda e\right)-J_{\mathrm{T} \alpha} \theta
$$

and replacing for $J_{\mathrm{T} \alpha} \theta$ from equation (5) yields

$$
J_{\mathrm{T} \alpha} \dot{r}=J_{\mathrm{T} \alpha}\left(\theta_{\mathrm{d}}+\lambda \dot{e}\right)+C_{\mathrm{T} \alpha} \theta-\tau_{\mathrm{m} \alpha}-\tau_{\mathrm{u}} \frac{\Delta_{\alpha} r_{\alpha}}{r_{\theta}}-\tau_{\mathrm{m} \beta} \frac{\Delta_{\alpha} r_{\alpha}}{\Delta_{\beta_{\beta}} r_{\beta}}
$$

which can be written as

$$
J_{\mathrm{T} \alpha} \dot{r}=\mathbf{Y}_{\alpha} \boldsymbol{\Phi}_{\alpha}-\tau_{\mathrm{m} \alpha}
$$

where $\mathbf{Y}_{\alpha}=\left[\left(\theta_{\mathrm{d}}+\lambda_{e}\right) \theta \tau_{\mathrm{u}} \tau_{\mathrm{m} \beta}\right]$ and selecting the control input, $\tau_{\mathrm{m} \alpha}$, as

$$
\tau_{\mathrm{m} \alpha}=\mathbf{Y}_{\alpha} \hat{\boldsymbol{\Phi}}_{\alpha}+K_{\mathrm{v} \alpha} r
$$

equation (15) may be written as

$$
\dot{V}_{\alpha}=-K_{\mathrm{v} \alpha} r^{2}+\boldsymbol{\Phi}_{\alpha}^{\mathrm{T}}\left(\mathbf{P}_{\alpha}^{-1} \dot{\boldsymbol{\Phi}}_{\alpha}+\mathbf{Y}_{\alpha}^{\mathrm{T}} r\right)+\frac{1}{2} \boldsymbol{\Phi}_{\alpha}^{\mathrm{T}} \dot{\mathbf{P}}_{\alpha}^{-1} \boldsymbol{\Phi}_{\alpha}
$$

The least-squares adaptive update law proposed by $\mathrm{Li}$ and Slotine [10], given by the following equations, is used here:

$$
\begin{aligned}
& \dot{\boldsymbol{\Phi}}_{\alpha}=\boldsymbol{\Phi}_{\alpha}-\dot{\boldsymbol{\Phi}}_{\alpha}=-\mathbf{P}_{\alpha} \mathbf{Y}_{\alpha}^{\mathrm{T}} r-\mathbf{P}_{\alpha} \mathbf{W}_{\mathrm{f} \alpha}^{\mathrm{T}} \tau_{\mathrm{f} \alpha} \\
& \dot{\mathbf{P}}_{\alpha}^{-1}=\mathbf{W}_{\mathrm{f} \alpha}^{\mathrm{T}} \mathbf{W}_{\mathrm{f} \alpha}
\end{aligned}
$$

Using equation (21) and replacing for $\tilde{\tau}_{\mathrm{fo}}$ from equations (12) and (13) and noting that $\boldsymbol{\Phi}_{\alpha}$ is a vector of constant parameters yields

$$
\dot{\boldsymbol{\Phi}}_{\alpha}=\mathbf{P}_{\alpha} \mathbf{Y}_{\alpha}^{\mathrm{T}} r+\mathbf{P}_{\alpha} \mathbf{W}_{\mathrm{f} \alpha}^{\mathrm{T}}\left(\tau_{\mathrm{f} \alpha}-\mathbf{W}_{\mathrm{f} \alpha} \boldsymbol{\Phi}_{\alpha}\right)
$$

Using the matrix identity given by

$$
\frac{\mathrm{d} \mathbf{A}^{-1}}{\mathrm{~d} t}=-\mathbf{A}^{-1} \frac{\mathrm{d} \mathbf{A}}{\mathrm{d} t} \mathbf{A}^{-1}
$$

equation (22) may be written more conveniently as

$$
\dot{\mathbf{P}}_{\alpha}=-\mathbf{P}_{\alpha} \mathbf{W}_{\mathrm{f} \alpha}^{\mathrm{T}} \mathbf{W}_{\mathrm{f} \alpha} \mathbf{P}_{\alpha}
$$

Therefore, it follows that

$$
\lim _{t \rightarrow \infty} \lambda_{\max }\left\{\mathbf{P}_{\alpha}\right\}=0
$$

and

$$
\lim _{t \rightarrow \infty} \lambda_{\min }\left\{\mathbf{P}_{\alpha}^{-1}\right\}=\infty
$$

where $\lambda_{\min }\{\mathbf{A}\}$ and $\lambda_{\max }\{\mathbf{A}\}$ represent the minimum and maximum eigenvalues of the matrix A. Equation (27) is referred to as the infinite integral condition. Substitution of the parameter update law and least-squares estimator, given by equations (22) and (23) respectively, into equation (20) leads to

$$
\dot{V}_{\alpha}=-K_{\mathrm{va} \alpha} r^{2}-\frac{1}{2} \boldsymbol{\Phi}_{\alpha}^{\mathrm{T}} \mathbf{W}_{\mathrm{f} \alpha}^{\mathrm{T}} \mathbf{W}_{\mathrm{f} \alpha} \boldsymbol{\Phi}_{\alpha}
$$




\section{$5.2 \beta$ unwinding control}

Similarly, for $\beta$ unwinding control, equation (1) can be used to solve for the $\beta$ motor torque by multiplying both sides by $\Delta_{\beta} r_{\beta} / r_{\theta}$, as given by

$$
\tau_{\mathrm{m} \beta}=J_{\mathrm{T} \beta} \dot{\theta}+C_{\mathrm{T} \beta} \theta-\tau_{\mathrm{u}} \frac{\Delta_{\beta} r_{\beta}}{r_{\theta}}-\tau_{\mathrm{m} \alpha} \frac{\Delta_{\beta} r_{\beta}}{\Delta_{\alpha} r_{\alpha}}=\mathbf{W}_{\beta} \boldsymbol{\Phi}_{\beta}
$$

where

$$
\begin{aligned}
& \mathbf{W}_{\beta}=\left[\begin{array}{llll}
\theta & \theta & \tau_{\mathrm{u}} & \tau_{\mathrm{m} \alpha}
\end{array}\right] \\
& \boldsymbol{\Phi}_{\beta}=\left[\begin{array}{llll}
J_{\mathrm{T} \beta} & C_{\mathrm{T} \beta} & -\frac{\Delta_{\beta} r_{\beta}}{r_{\theta}}-\frac{\Delta_{\beta} r_{\beta}}{\Delta_{\alpha} r_{\alpha}}
\end{array}\right]^{\mathrm{T}}
\end{aligned}
$$

Following the method outlined above, the control input, $\tau_{\mathrm{m} \beta}$, can be selected as

$$
\tau_{\mathrm{m} \beta}=\mathbf{Y}_{\beta} \hat{\boldsymbol{\Phi}}_{\beta}+K_{\mathrm{v} \beta} r
$$

The least-squares adaptive update law is given by

$$
\begin{aligned}
& \dot{\boldsymbol{\Phi}}_{\beta}=-\dot{\boldsymbol{\Phi}}_{\beta}=-\mathbf{P}_{\beta} \mathbf{Y}_{\beta}^{\mathrm{T}} r-\mathbf{P}_{\beta} \mathbf{W}_{\mathrm{f} \beta}^{\mathrm{T}} \tau_{\mathrm{f} \beta} \\
& \dot{\boldsymbol{\Phi}}_{\beta}=\mathbf{P}_{\beta} \mathbf{Y}_{\beta}^{\mathrm{T}} r+\mathbf{P}_{\beta} \mathbf{W}_{\mathrm{f} \beta}^{\mathrm{T}}\left(\tau_{\mathrm{f} \beta}-\mathbf{W}_{\mathrm{f} \beta} \hat{\boldsymbol{\Phi}}_{\beta}\right) \\
& \dot{\mathbf{P}}_{\beta}^{-1}=\mathbf{W}_{\mathrm{f} \beta}^{\mathrm{T}} \mathbf{W}_{\mathrm{f} \beta} \\
& \dot{\mathbf{P}}_{\beta}=-\mathbf{P}_{\beta} \mathbf{W}_{\mathrm{f} \beta}^{\mathrm{T}} \mathbf{W}_{\mathrm{f} \beta} \mathbf{P}_{\beta}
\end{aligned}
$$

and the time derivative of the Lyapunov function is given by

$$
\dot{V}_{\beta}=-K_{\mathrm{v}} \beta^{r^{2}-\frac{1}{2}} \boldsymbol{\Phi}_{\beta}^{\mathrm{T}} \mathbf{W}_{\mathrm{f} \beta}^{\mathrm{T}} \mathbf{W}_{\mathrm{f} \beta} \boldsymbol{\Phi}_{\beta}
$$

\subsection{Stability analysis}

The Lyapunov functions for $\alpha$ and $\beta$ unwinding may be written as

$$
V_{i}=\frac{1}{2} J_{\mathrm{T} i} r^{2}+\frac{1}{2} \boldsymbol{\Phi}_{i}^{\mathrm{T}} \mathbf{P}_{i}^{-1} \boldsymbol{\Phi}_{i}
$$

where the subscript $i$ should be replaced by $\alpha$ for $\alpha$ unwinding or $\beta$ for $\beta$ unwinding. Similarly, the time derivative of the Lyapunov function, given by equation (34), can also be expressed as

$$
\dot{V}_{i}=-K_{\mathrm{vi}} r^{2}-\frac{1}{2} \boldsymbol{\Phi}_{i}^{\mathrm{T}} \mathbf{W}_{\mathrm{f} i}^{\mathrm{T}} \mathbf{W}_{\mathrm{f} i} \boldsymbol{\Phi}_{i} \leqslant 0
$$

By showing that the second derivative of the Lyapunov function is bounded, so proving that $\dot{V}_{i}$ is uniformly continuous, Barbalat's lemma can be invoked to show that the tracking error, $r$, and parameter error, $\boldsymbol{\Phi}_{i}$, both converge to zero. Barbalat's lemma states that if $\dot{x}(t)$ is a uniformly continuous, real function and $\lim _{t \rightarrow \infty} x(t)=k<\infty$, then $\lim _{t \rightarrow \infty} \dot{x}(t)=0$ [11]. Firstly, $\ddot{V}_{i}$ is obtained by differentiating equation (35) to give

$$
\ddot{V}_{i}=-2 K_{\mathrm{v} i} r \dot{r}-\boldsymbol{\Phi}_{i}^{\mathrm{T}} \mathbf{W}_{\mathrm{f} i}^{\mathrm{T}} \mathbf{W}_{\mathrm{f} i} \dot{\boldsymbol{\Phi}}_{i}-\boldsymbol{\Phi}_{i}^{\mathrm{T}} \mathbf{W}_{\mathrm{f} i}^{\mathrm{T}} \mathbf{W}_{\mathrm{f} i} \boldsymbol{\Phi}_{i}
$$

Therefore, in order to prove that $\ddot{V}_{i}$ is bounded and $\dot{V}_{i}$ is uniformly continuous, it is necessary and sufficient to prove that $r, \dot{r}, \boldsymbol{\Phi}_{i}, \dot{\boldsymbol{\Phi}}_{i}, \mathbf{W}_{\mathrm{f} i}$ and $\dot{\mathbf{W}}_{\mathrm{f} i}$ are bounded. $\dot{V}_{i}$ is at least negative semi-definite, implying that $V_{i} \leqslant V_{i}(0)$ and $r$ and $\boldsymbol{\Phi}_{i}$ are bounded. Subsequently, from the definition of $r$ in equation (4), $e$ and $\dot{e}$ are also bounded. Considering $\mathbf{Y}_{i}$ as defined in equation (18), i.e. $\mathbf{Y}_{i}=$ $\left[\begin{array}{llll}\theta_{\mathrm{d}}+\lambda_{e} & \theta & \tau_{\mathrm{u}} & \tau_{\mathrm{m} i}{ }^{\prime}\end{array}\right]$, where $i^{\prime}$ should be replaced by $\beta$ for $\alpha$ unwinding and $\alpha$ for $\beta$ unwinding, and assuming that the inputs, $\tau_{\mathrm{u}}, \tau_{\mathrm{m} i^{\prime}}, \theta_{\mathrm{d}}, \theta_{\mathrm{d}}$ and $\theta_{\mathrm{d}}$, are bounded, then $\theta$, $\theta$ and $\mathbf{Y}_{i}$ must also be bounded. Substitution of the control law (19) or (30) into equation (18) leads to

$$
J_{\mathrm{T} i} \dot{r}=\mathbf{Y}_{i} \boldsymbol{\Phi}_{i}-K_{\mathrm{v} i} r
$$

Noting that $J_{\mathrm{T} i}>0$, then $\dot{r}$ and $\theta$ are also bounded. Subsequently, from equation (5) or (29) and from the assumption that $\boldsymbol{\Phi}_{i}$ is bounded, $\tau_{\mathrm{m} i}, \mathbf{W}_{i}$ and $\hat{\boldsymbol{\Phi}}_{i}$ are bounded. From the definition of the filter, $f$, it can be seen that $\tau_{\mathrm{f} \alpha}, \mathbf{W}_{\mathrm{f} i}$ and $\dot{\mathbf{W}}_{\mathrm{f} i}$ are also bounded. Finally, to prove that $\ddot{V}_{i}$ is bounded the parameter update law of equation (23) or (31) may be written as

$$
\dot{\boldsymbol{\Phi}}_{i}=-\mathbf{P}_{i} \mathbf{Y}_{i}^{\mathrm{T}} r-\mathbf{P}_{i} \mathbf{W}_{\mathrm{f} i}^{\mathrm{T}} \tilde{t}_{\mathrm{f} i}
$$

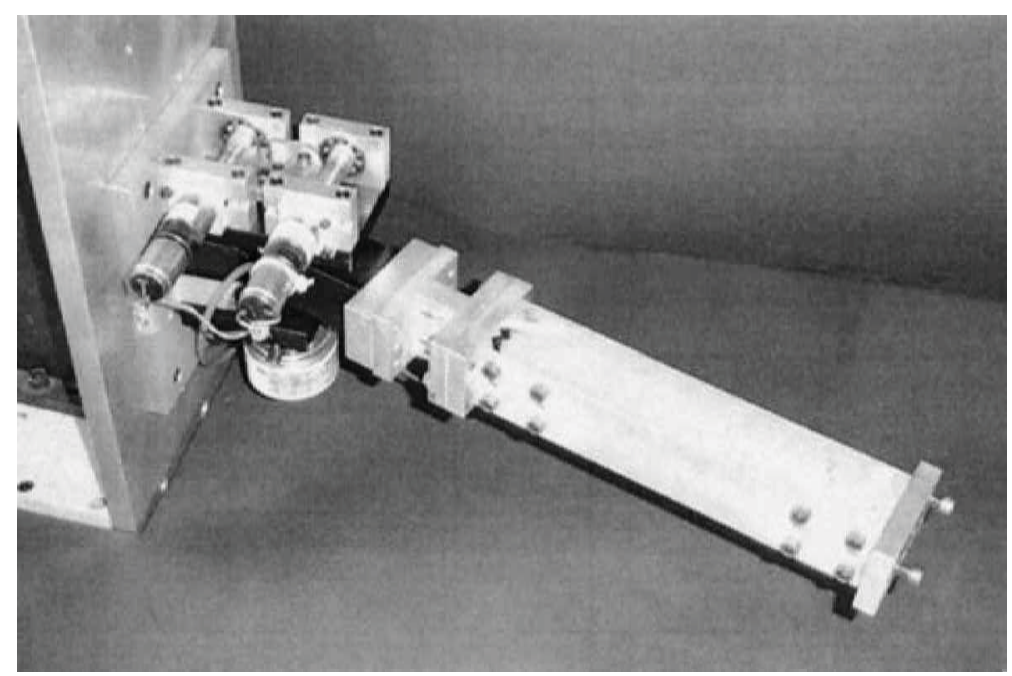

Fig. 3 Worm driven joint 


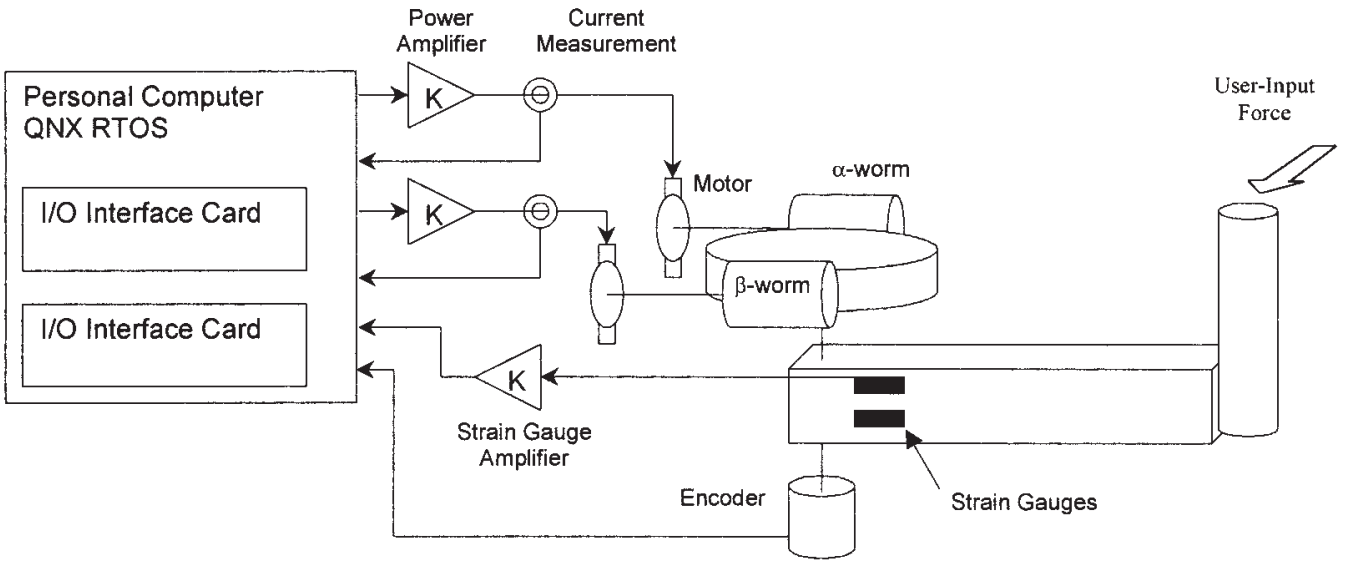

Fig. 4 Schematic overview of the control system
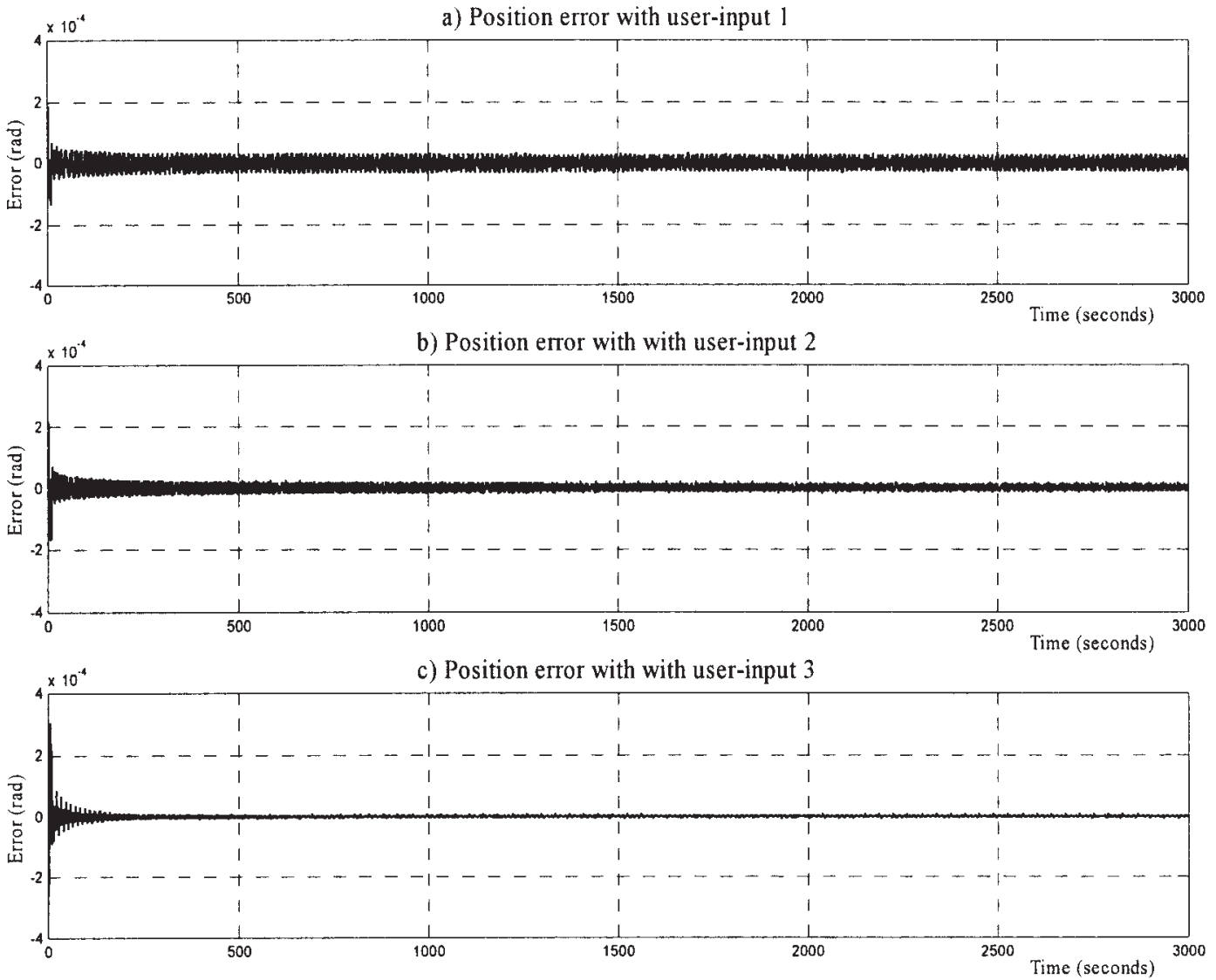

Fig. 5 Comparison of tracking errors during the simulation of the three user-input torque commands

From equations (22) or (32), $\mathbf{P}_{i}$ is bounded by $\mathbf{P}_{i}(0)$ since $\dot{\mathbf{P}}_{i}$ is negative semi-definite. From equations (12) and (13) and the fact that the filter, $f$, has a stable transfer function, it can be seen that $\dot{\boldsymbol{\Phi}}_{i}$ is bounded. Application of Barbalat's lemma yields

$$
\lim _{t \rightarrow \infty} \dot{V}_{i}=0
$$

and

$$
\lim _{t \rightarrow \infty} r=0
$$

I06701 @ IMechE 2002
Since the relationship between $r$ and $e$, given in equation (4), may also be written in terms of the strictly proper, asymptotically stable transfer function, $H(s)$, such that

$$
e(s)=H(s) r(s)
$$

it can be concluded that

$$
\lim _{t \rightarrow \infty} r=0 \Rightarrow \lim _{t \rightarrow \infty} e=0
$$

It is also possible to define the type of stability for the Proc Instn Mech Engrs Vol 216 Part I: J Systems and Control Engineering 


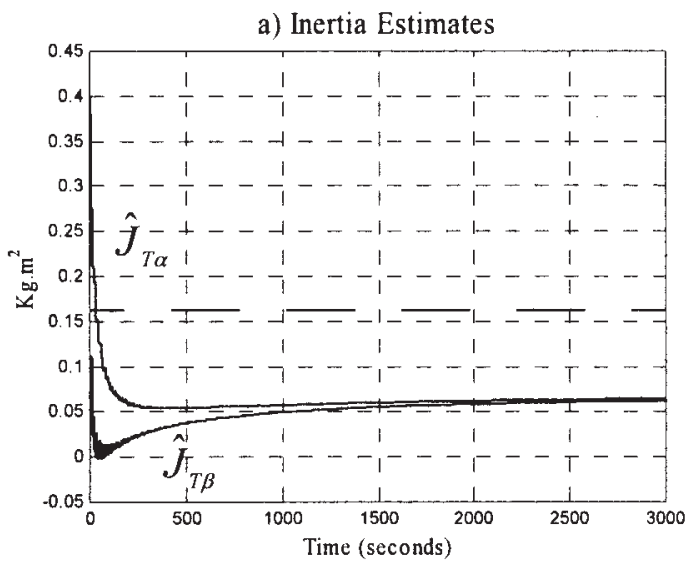

c) User-input Torque Coefficient Estimates

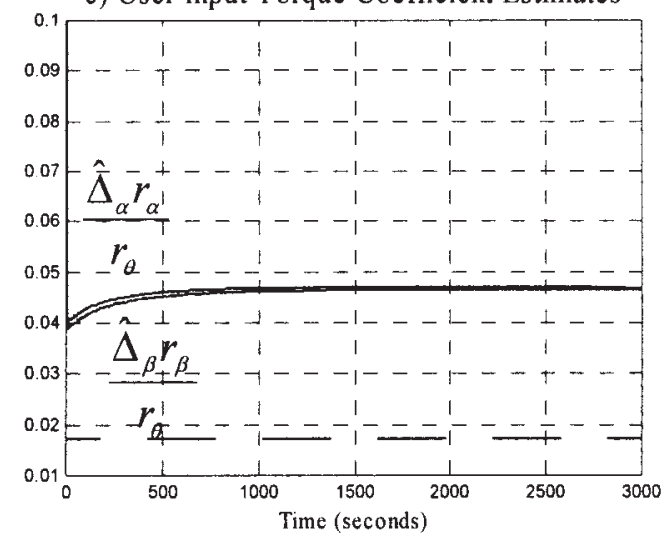

b) Damping Coefficient Estimates

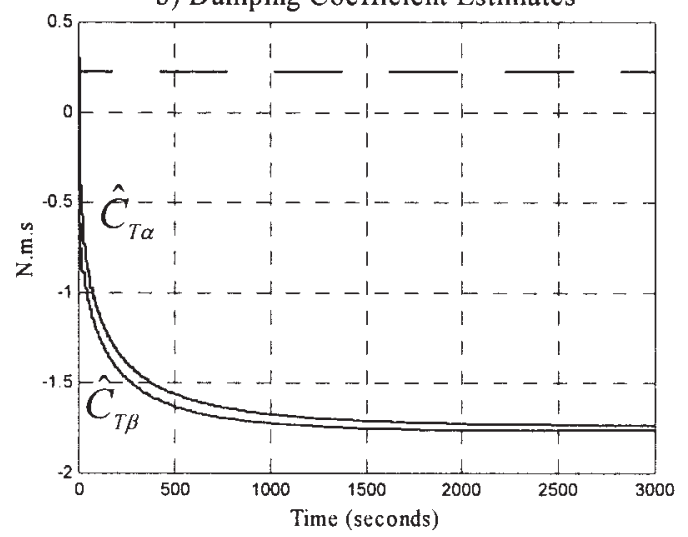

d) Motor Torque Coefficient Estimates

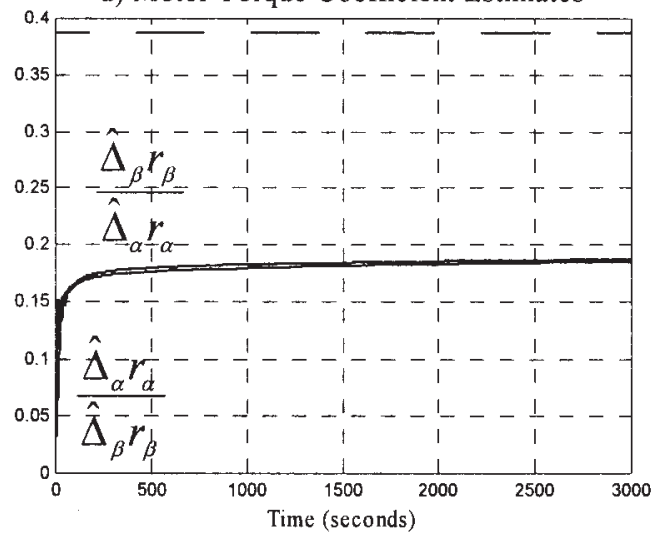

Fig. 6 Simulation parameter estimates with user-input 1

parameter error. Since $V_{i}$ is bounded by $V_{i}(0)$, if the infinite integral condition given in equation (27) holds, it must be concluded that the parameter error tends to zero, i.e. $\lim _{t \rightarrow \infty} \tilde{\Phi}_{i}=0$. The infinite integral condition is less restrictive than the persistency of excitation condition discussed above, owing to the fact that as long as there is input to the system and that the system is capable of motion it is easy to prove that equation (27) holds true. A persistently exciting trajectory/input, on the other hand, is not always easy to derive or generate in most robotic applications.

\section{EXPERIMENTAL SET-UP}

Figure 3 shows the dual-worm driven joint mechanism. The joint is controlled using a Pentium $233 \mathrm{MHz}$ personal computer running the QNX 4.25 real-time operating system [12]. A schematic diagram of the control system is shown in Fig. 4.

The control algorithm requires measurements of motor torque, user-input force and position. The userinput force is measured using four strain gauges, in a Wheatstone bridge configuration, mounted on a specially designed section of the link. Closed-loop regu- lation of the motor output torque is accomplished by measuring the armature current and implementing a digital PI (proportional + integral) controller. All analogue signals are measured and generated using 12-bit analogue-to-digital converters and 12-bit digitalto-analogue converters. The link rotation is recorded using an encoder and appropriate electronics to generate 20000 counts per revolution, giving a resolution of $0.0003142 \mathrm{rad} /$ count (or $0.018 \%$ count). The encoder position is read as a 24-bit number from an HCTL-1100 motion control interface. The frequency of the control loop is set at $600 \mathrm{~Hz}$ and link rotational velocity measurement is obtained in software by using a backward difference algorithm.

\section{RESULTS AND DISCUSSION}

\subsection{Simulation results}

The mathematical model of the dual worm-driven joint was simulated using SIMULINK. The parameters of the model were set according to Table 1 and the control parameters set as follows: tracking error filter gain $\lambda=50$ and controller gains $K_{\mathrm{v} \alpha}=K_{\mathrm{v} \beta}=20$. The dynamic effects 


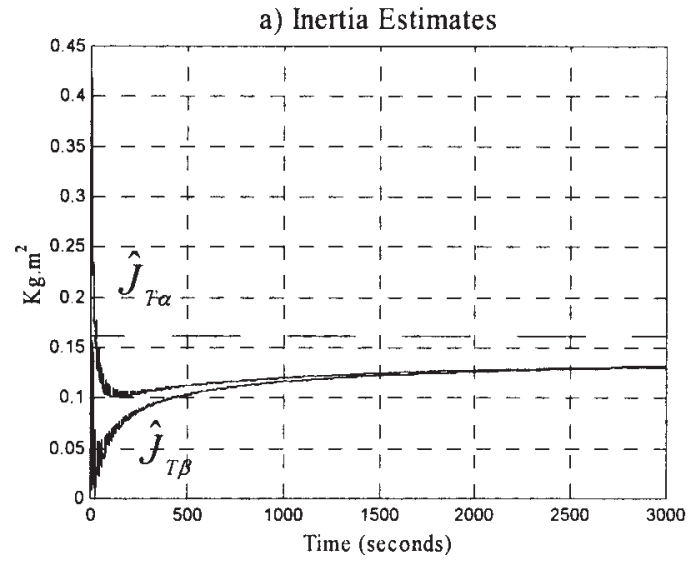

c) User-input Torque Coefficient Estimates

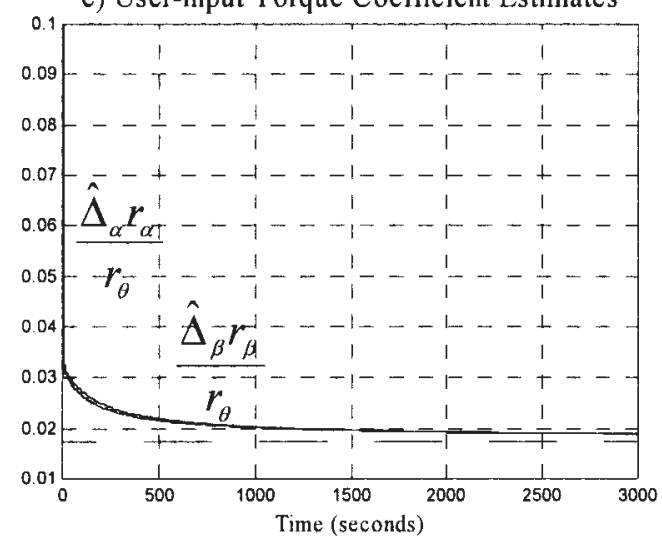

b) Damping Coefficient Estimates

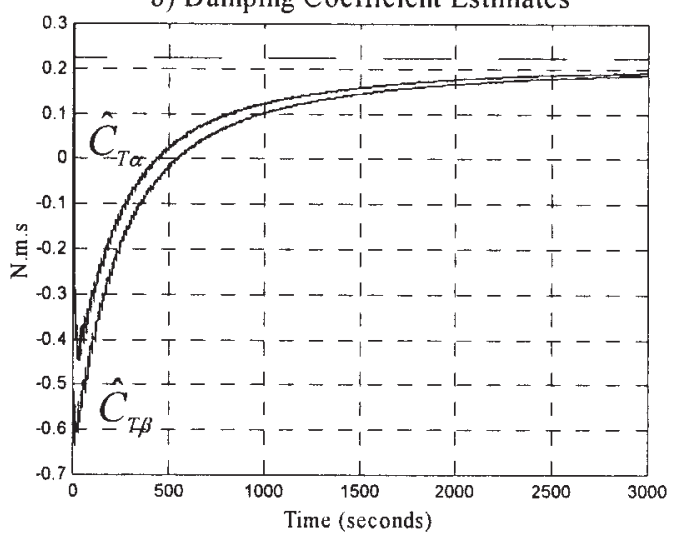

d) Motor Torque Coefficient Estimates

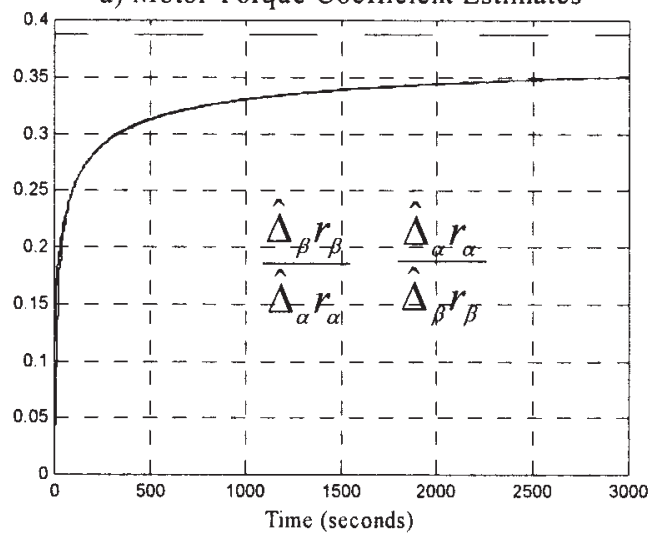

Fig. 7 Simulation parameter estimates with user-input 2

of the $\alpha$ and $\beta$ motors are cancelled using a high-gain PI torque control feedback loop, assuming that motor output is measured without error. During the simulation the $\beta$ motor is set to follow the worm with constant torque under $\alpha$ unwinding control. Similarly, during $\beta$ unwinding the $\alpha$ motor is also set to follow the wormwheel with constant torque. The matrices $\mathbf{P}_{\alpha}$ and $\mathbf{P}_{\beta}$ were chosen to give well-damped parameter estimates, and the parameter estimates themselves, $\hat{\boldsymbol{\Phi}}_{\alpha}$ and $\hat{\boldsymbol{\Phi}}_{\beta}$, were initialized with arbitrary values according to

$$
\begin{gathered}
\mathbf{P}_{\alpha}(0)=\mathbf{P}_{\beta}(0)=\left[\begin{array}{cccc}
100 & 0 & 0 & 0 \\
0 & 1000 & 0 & 0 \\
0 & 0 & 1 & 0 \\
0 & 0 & 0 & 10
\end{array}\right] \\
\boldsymbol{\Phi}_{\alpha}(0)=\left[\begin{array}{l}
0.4 \\
0.1 \\
0.1 \\
0.5
\end{array}\right], \\
\boldsymbol{\Phi}_{\beta}(0)=\left[\begin{array}{l}
0.1 \\
0.3 \\
0.1 \\
0.1
\end{array}\right]
\end{gathered}
$$

Torque filtering was performed using the linear filter I06701 $\odot$ IMechE 2002 given by the first-order transfer function

$$
f(s)=\frac{1}{s+1}
$$

The same filter was used in both the $\alpha$ unwinding and $\beta$ unwinding controllers.

Three user-input torque commands, $\tau_{\mathrm{u} 1}, \tau_{\mathrm{u} 2}$ and $\tau_{\mathrm{u} 3}$, given by the following equations respectively, were applied to the model:

$$
\begin{aligned}
\tau_{\mathrm{u} 1} & =5 \tanh [\cos (4 t)-0.76152] \operatorname{sgn}[\sin (t / 2)] \\
\tau_{\mathrm{u} 2} & =8 \tanh [\cos (4 t)-0.76152] \operatorname{sgn}[\sin (t / 2)] \\
\tau_{\mathrm{u} 3} & =8[\cos (4 t)+1.5] \sin (t / 2)
\end{aligned}
$$

The desired velocity for the system is calculated using equation (2) with $\theta_{\mathrm{d}, \max }=0.1 \mathrm{rad} / \mathrm{s}, \tau_{\mathrm{u}, \max }=10 \mathrm{~N} \mathrm{~m}$ and $\tau_{\mathrm{u}, \min }=1 \mathrm{Nm}$. With the system parameters given by Table 1 , the actual parameter vectors $\boldsymbol{\Phi}_{\alpha}$ and $\boldsymbol{\Phi}_{\beta}$ can be calculated for $|\theta| \neq 0$, as given by

$$
\boldsymbol{\Phi}_{\alpha}=\boldsymbol{\Phi}_{\beta}=\left[\begin{array}{l}
0.162 \\
0.223 \\
0.017 \\
0.387
\end{array}\right]
$$


Table 1 Simulation model parameters

\begin{tabular}{lll}
\hline & Worm-wheel & $\alpha$ and $\beta$ worm \\
\hline Inertia & $J_{\theta}=0.25 \mathrm{~kg} \mathrm{~m}^{2}$ & $J_{\alpha}=J_{\beta}=0.003698 \mathrm{~kg} \mathrm{~m}^{2}$ \\
Viscous damping & $C_{\mathrm{f} \theta}=0.005 \mathrm{~N} \mathrm{~m} \mathrm{~s} / \mathrm{rad}$ & $C_{\mathrm{f} \alpha}=C_{\mathrm{f}}=0.005 \mathrm{~N} \mathrm{~m} \mathrm{~s} / \mathrm{rad}$ \\
Gear geometry & $r_{\theta}=0.025 \mathrm{~m}$ & $r_{\alpha}=r_{\beta}=0.0065 \mathrm{~m}$ \\
Static friction & & $\mu_{\mathrm{s} \alpha}=\mu_{\mathrm{s} \beta}=0.14$ \\
Dynamic friction & & $\mu_{\mathrm{d} \alpha}=\mu_{\mathrm{d} \beta}=0.12$ \\
Friction constant & $\varepsilon=0.001$ \\
Worm lead angle & $\gamma=0.05236 \mathrm{rad}$ \\
\hline
\end{tabular}

Figure 5 shows the position error recorded during the simulations for each user-input. The various rates at which the magnitude of the tracking error converges to zero can be clearly seen. Figures 6, 7 and 8 show the parameter estimates of the simulated system with the user-inputs defined by equations (45), (46) and (47) respectively. The expected values of the parameters are shown by dotted lines. Figure 6 illustrates that for the first user-input command [given by equation (45)] the estimated parameters do not converge on the expected values given by equation (48). However, with the two user-input commands given by equations (46) and (47), the parameter error converges to zero. The input given by equation (46) yields slower convergence of the parameter estimates to the true values than the input of equation (47), as shown in Figs 7 and 8 respectively.

It should be noted that the magnitude of the userinput force affects the convergence of the parameters. The problem arises from the choice of $\theta_{d}$ in equation (2). With the user-input defined by equation (45) the desired velocity function never saturates, i.e. $\theta_{\mathrm{d}}<\theta_{\mathrm{d} \text {,max }}$. Thus the dependence of $\theta_{\mathrm{d}}$ on $\tau_{\mathrm{u}}$ is very strong and as inputs they appear to be very similar. Under these conditions the composite adaptive controller cannot extract enough information from these inputs to determine the correct parameter estimates. However, the parameter estimates and tracking error remain bounded at all times.

The user-inputs given by equations (45) and (46) differ only in magnitude. This difference significantly changes the appearance of $\theta_{\mathrm{d}}$ with respect to $\tau_{\mathrm{u}}$ in the region where $\left|\tau_{\mathrm{u}}\right|>\tau_{\mathrm{u} \text {,max }}$. The composite adaptive controller is able to extract more information about the system with the user-inputs defined by equations (46) and (47). These effects must be taken into account during experimental work.
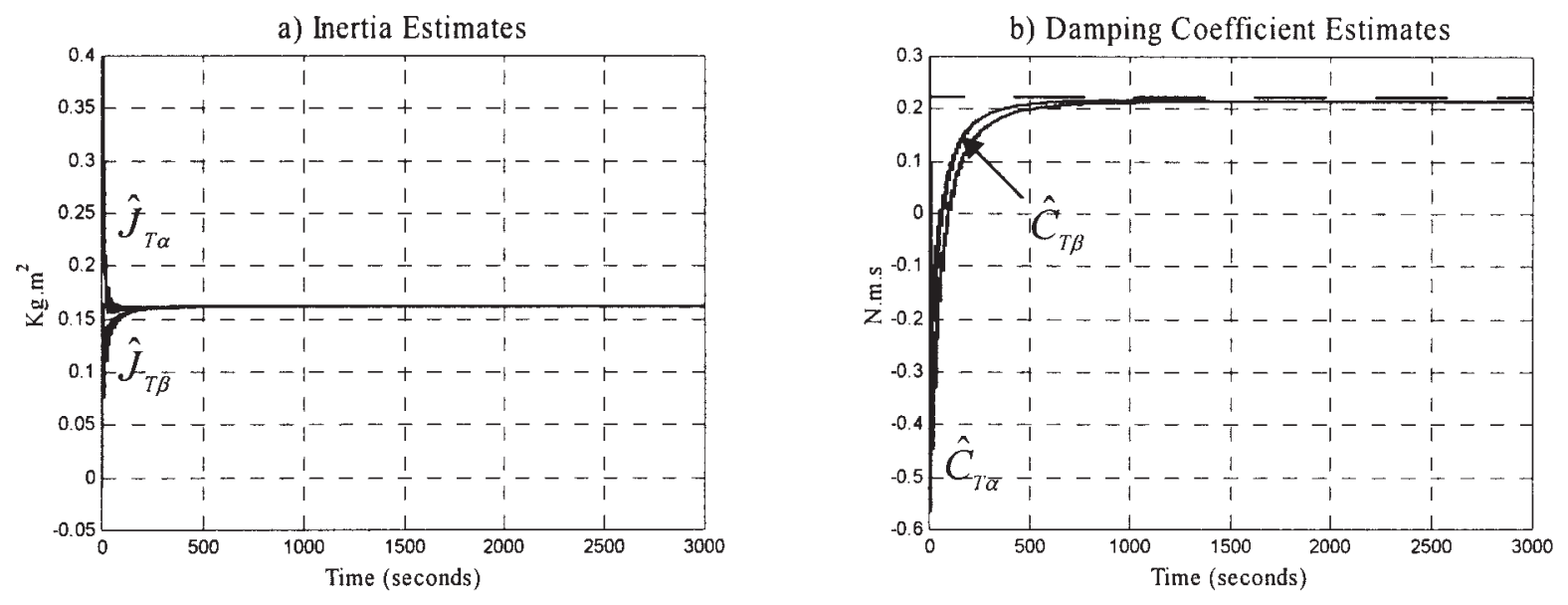

c) User-input Torque Coefficient Estimates

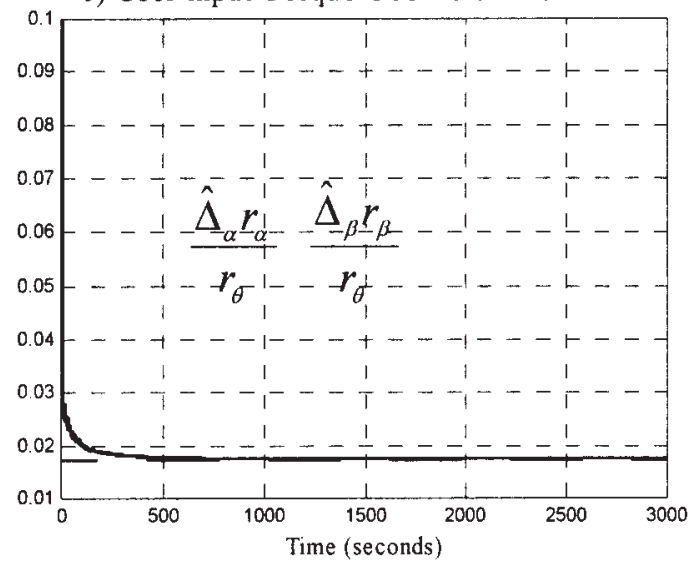

d) Motor Torque Coefficient Estimates

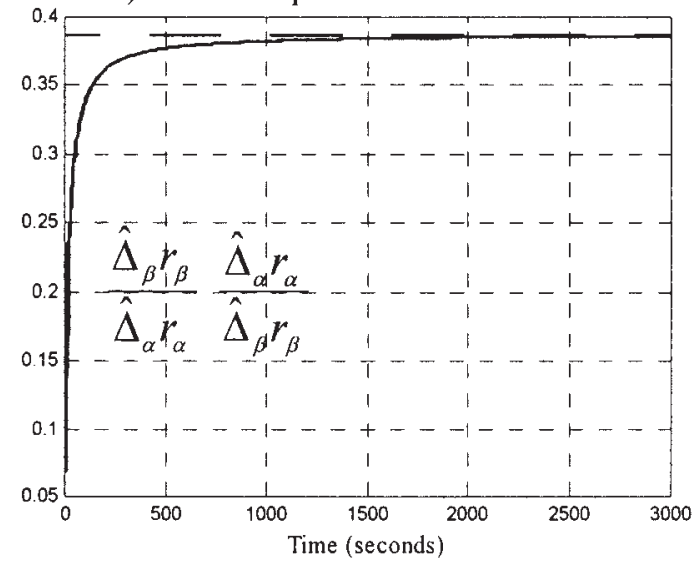

Fig. 8 Simulation parameter estimates with user-input 3 
a) Experimental user-input torque 1

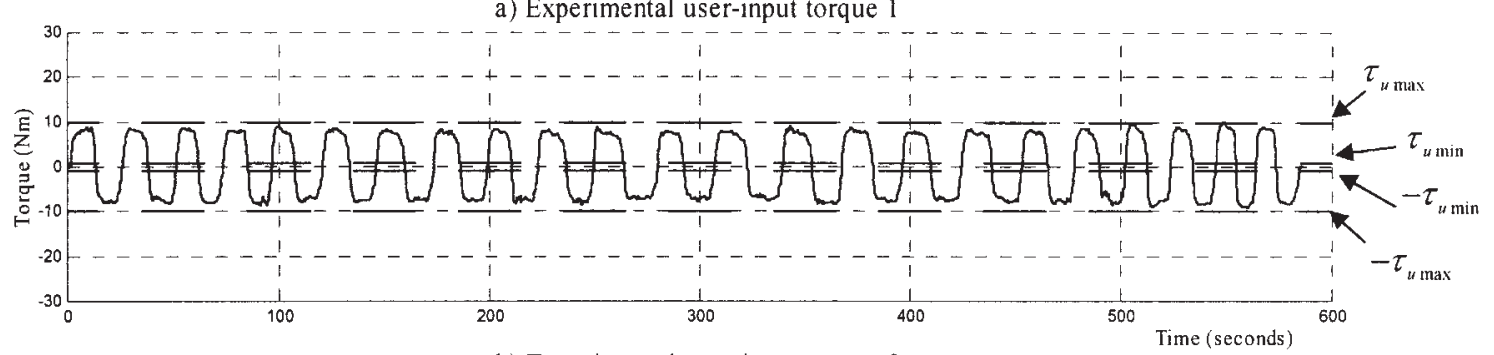

b) Experimental user-input torque 2

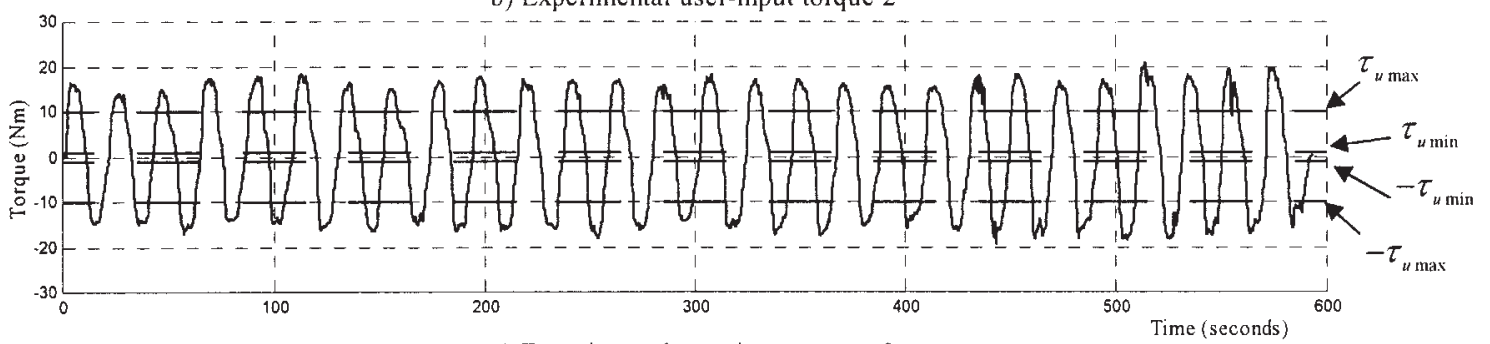

c) Experimental user-input torque 3

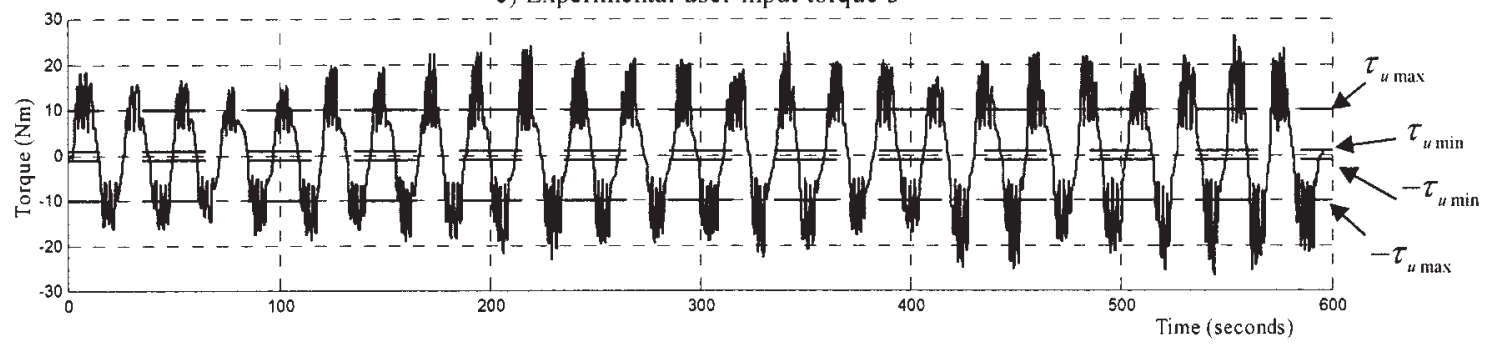

Fig. 9 Experimental user-input torque commands

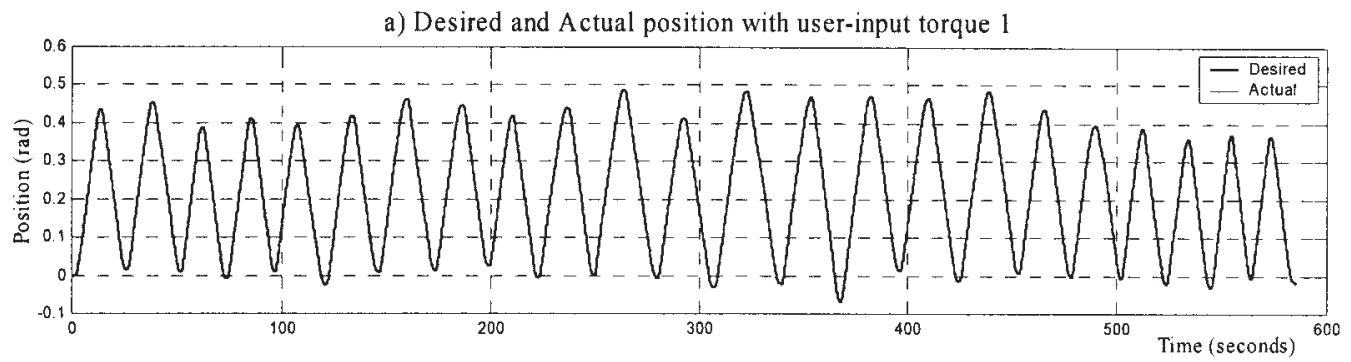

b) Desired and Actual position with user-input torque 2

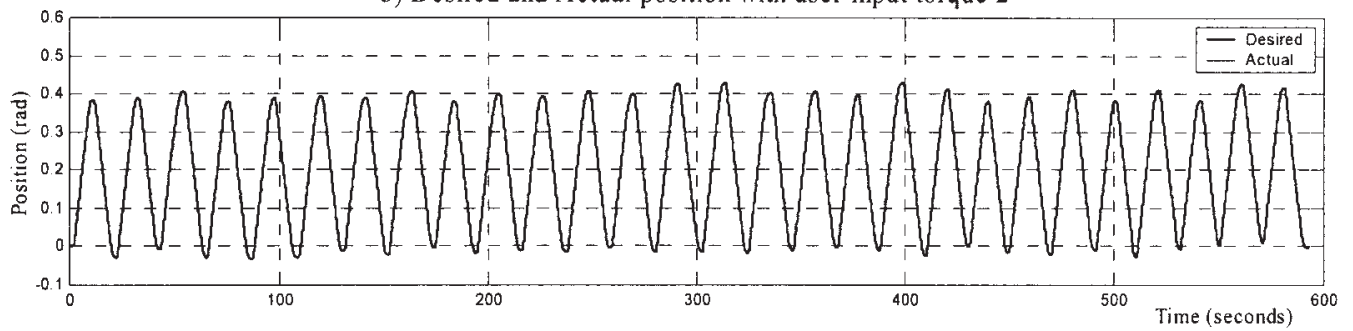

c) Desired and Actual position with user-input torque 3

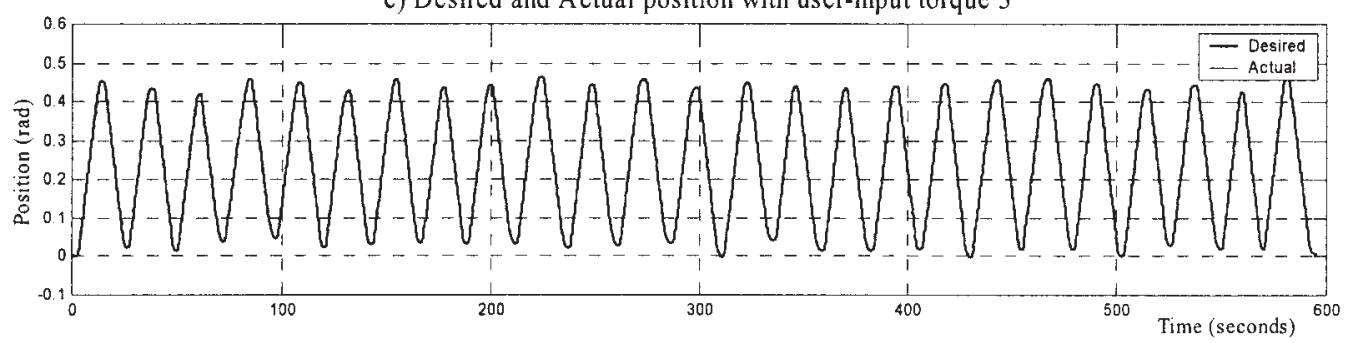

Fig. 10 Experimental desired and actual positions (note that the desired and actual positions are indistinguishable because the error is very small, as shown in Fig. 11) 
a) Error with user-input torque 1

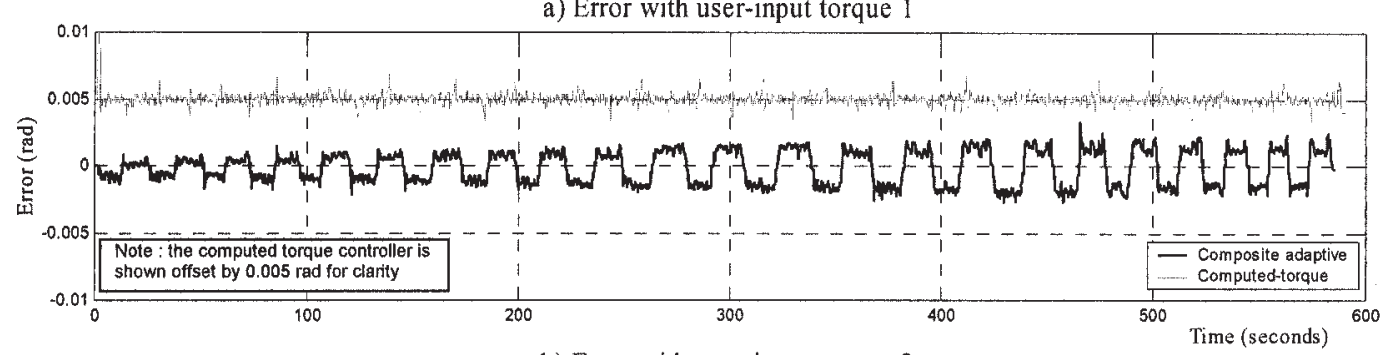

b) Error with user-input torque 2

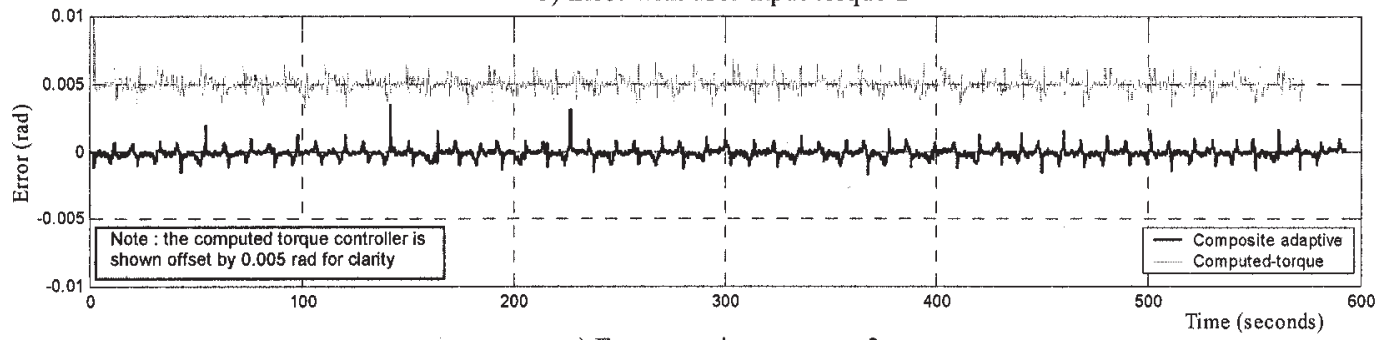

c) Error user-input torque 3

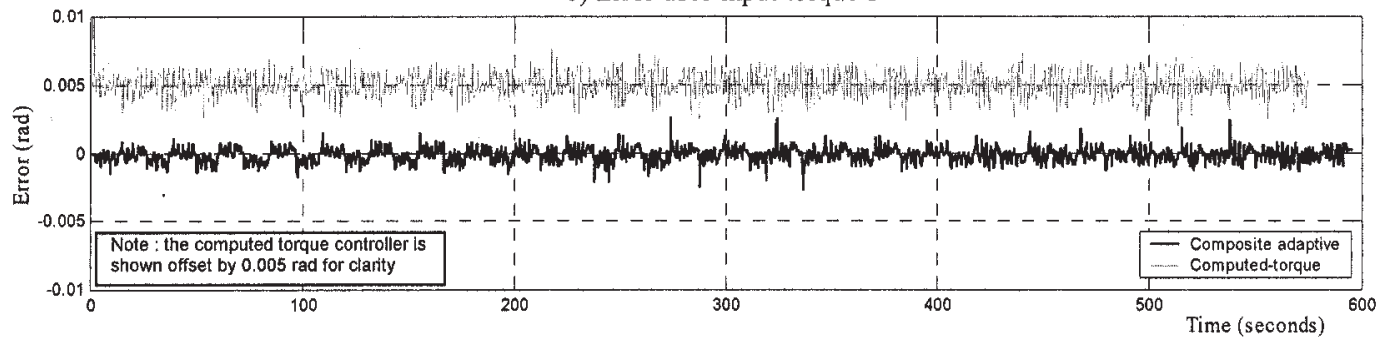

Fig. 11 Computed-torque control versus composite adaptive control

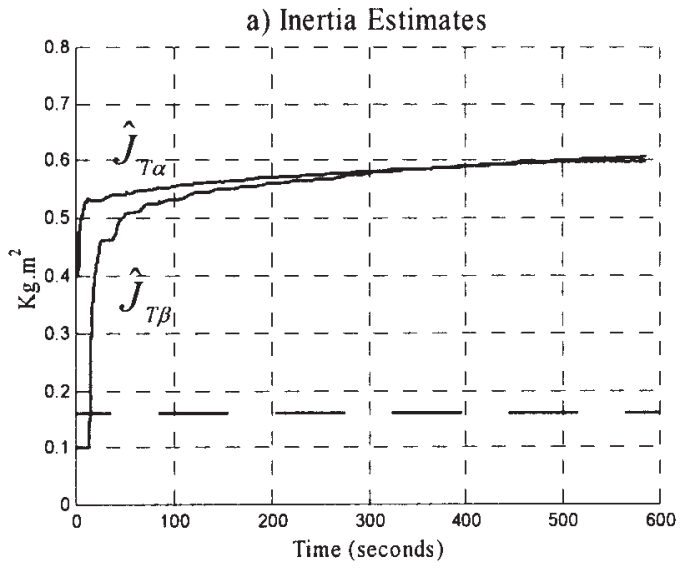

c) User-input Torque Coefficient Estimates

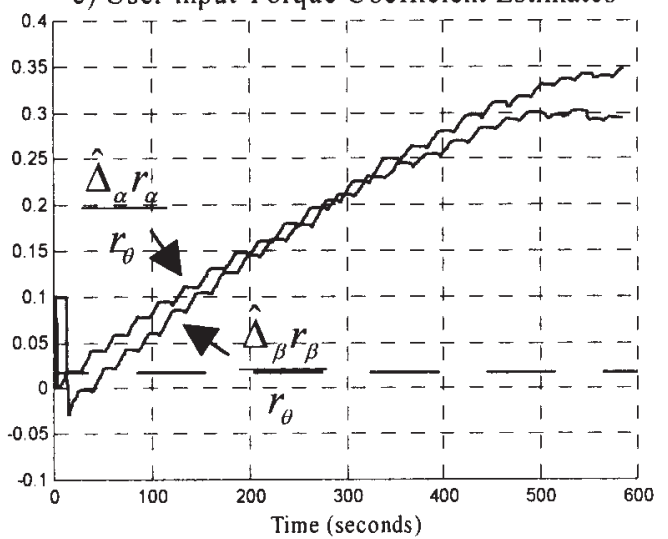

b) Damping Coefficient Estimates

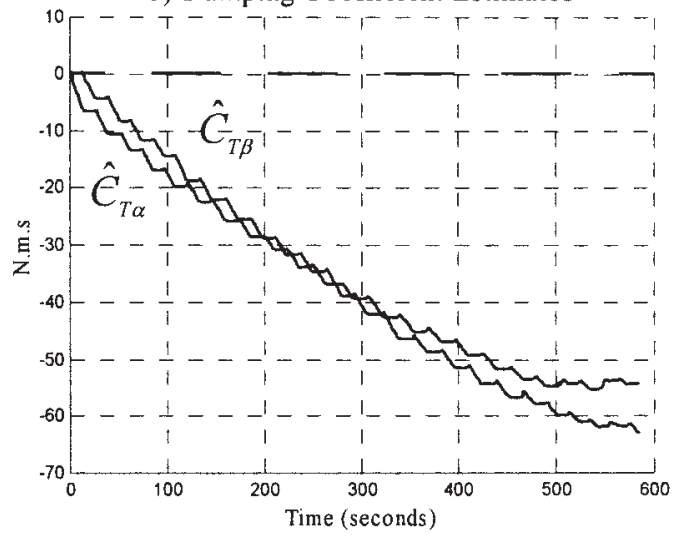

d) Motor Torque Coefficient Estimates

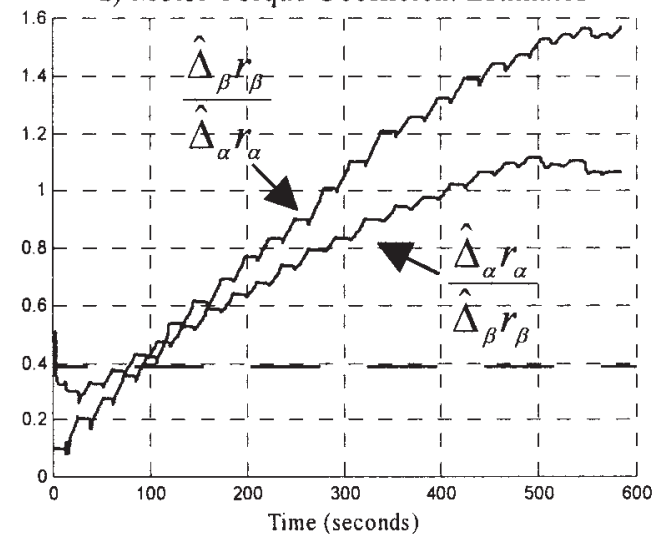

Fig. 12 Experimental parameter estimates with user-input 1 


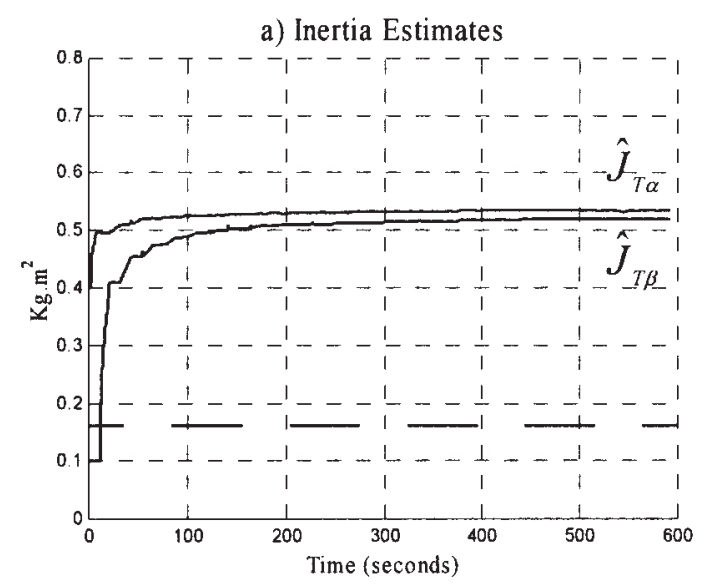

c) User-input Torque Coefficient Estimates

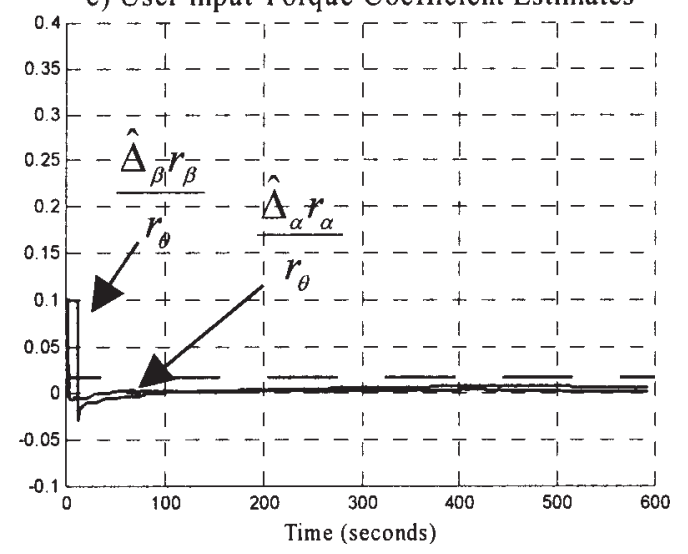

b) Damping Coefficient Estimates

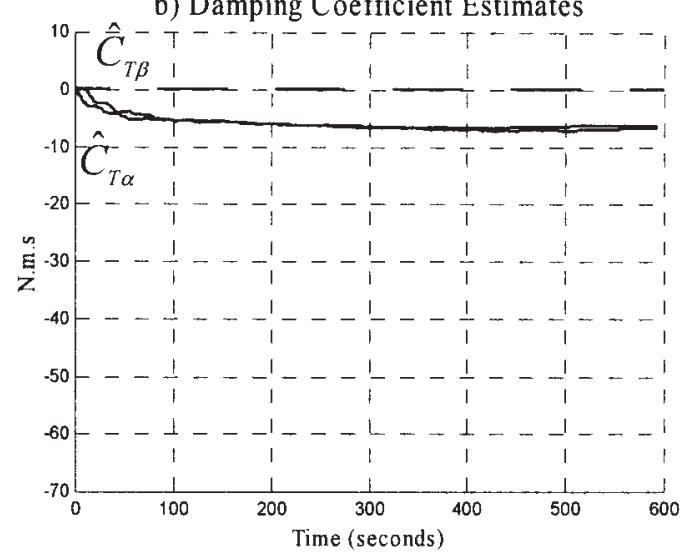

d) Motor Torque Coefficient Estimates

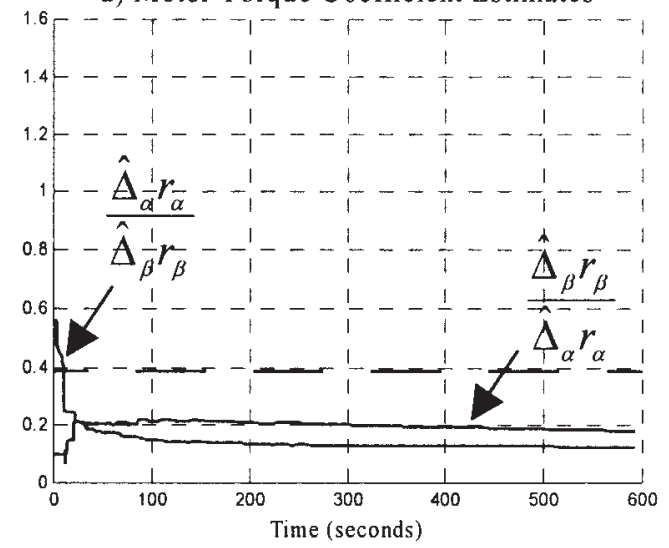

Fig. 13 Experimental parameter estimates with user-input 2

\subsection{Experimental results and discussion}

The experimental results of the computed-torque controller are compared to the results obtained from the composite adaptive controller. In both experiments the applied user-input force generates a command velocity based on equation (2), where $\theta_{\text {d,max }}=0.05 \mathrm{rad} / \mathrm{s}$, $\tau_{\mathrm{u}, \max }=10 \mathrm{~N} \mathrm{~m}, \tau_{\mathrm{u}, \min }=1 \mathrm{~N} \mathrm{~m}$. The desired velocity is then digitally integrated and differentiated to give the desired position and desired acceleration respectively. The matrices $\mathbf{P}_{\alpha}$ and $\mathbf{P}_{\beta}$ and the vectors $\hat{\boldsymbol{\Phi}}_{\alpha}$ and $\hat{\boldsymbol{\Phi}}_{\beta}$ were initialized as in equation (43) and the controller gains set as follows: tracking error filter gain $\lambda=50$ and controller gains $K_{\mathrm{v} \alpha}=K_{\mathrm{v} \beta}=35$. The torque filter that was used in the experiments for both $\alpha$ unwinding and $\beta$ unwinding control is given by the first-order transfer function of

$$
f(s)=\frac{10}{s+10}
$$

The filter of equation (49) used in these experiments differs from the filter used in the simulation tests [given in equation (44)] because it has been tuned to minimize the effects of quantization and sensor noise that are not modelled in the simulation.

In a similar manner to the simulation tests, three userinput torque commands were manually applied to the joint mechanism, as shown by Fig. 9. Figures 9a, b and c show the user-input commands similar to those given in equations (45), (46) and (47) respectively. Figure 10 shows the desired and actual position, derived from the integration of equation (2), for the three user-input torque commands. The graphs of desired and actual positions in each are indistinguishable because the error is very small.

The system position error using the composite adaptive and computed-torque controllers is shown in Fig. 11. The error has been shown using a small scale, compared to Fig. 10, to enlarge the graph. Although it has not been possible to generate exactly the same trajectory, Fig. 11 shows a comparison between the composite adaptive control scheme developed in this paper and the computed-torque method developed previously [5]. Figure 11a shows the performance of the computedtorque controller compared to that of the composite adaptive controller (with the user-input command of 


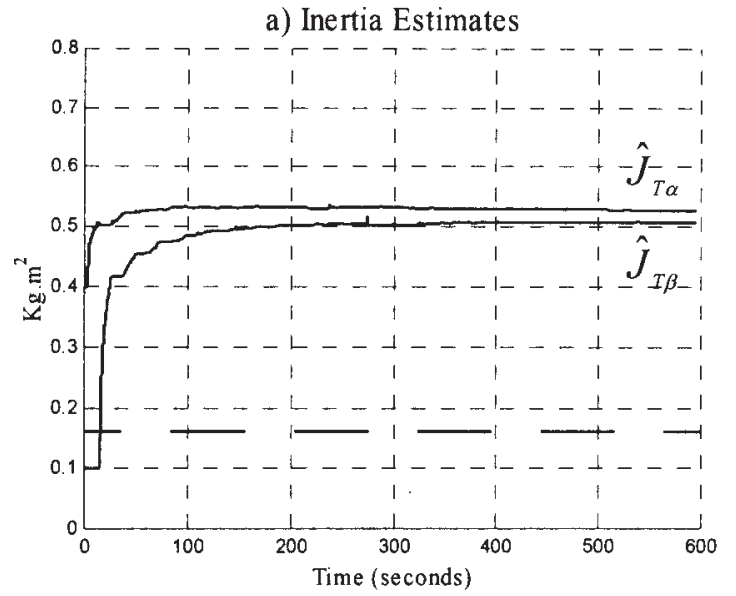

c) User-input Torque Coefficient Estimates

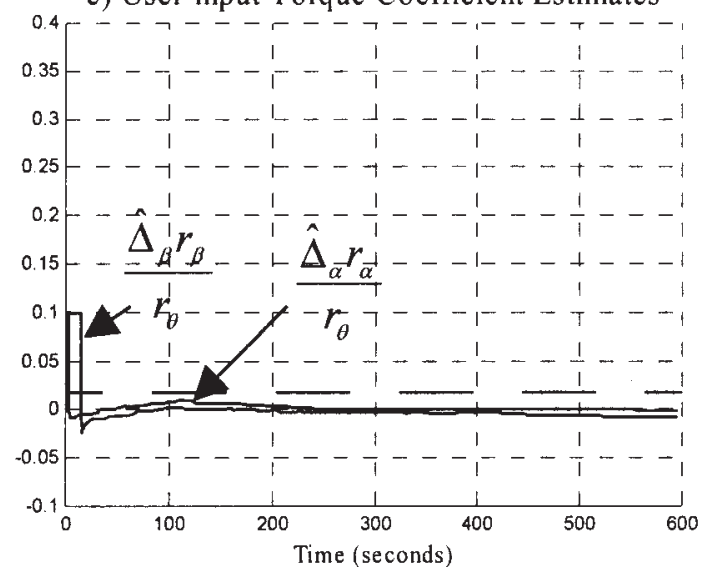

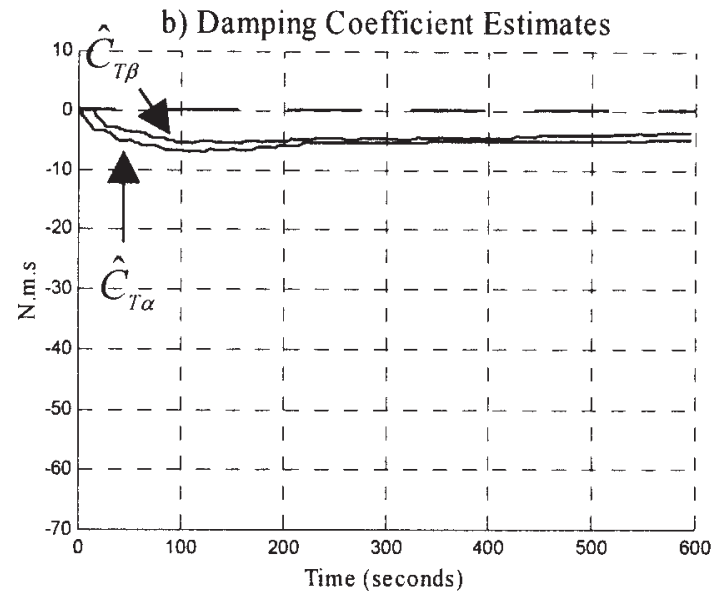

d) Motor Torque Coefficient Estimates

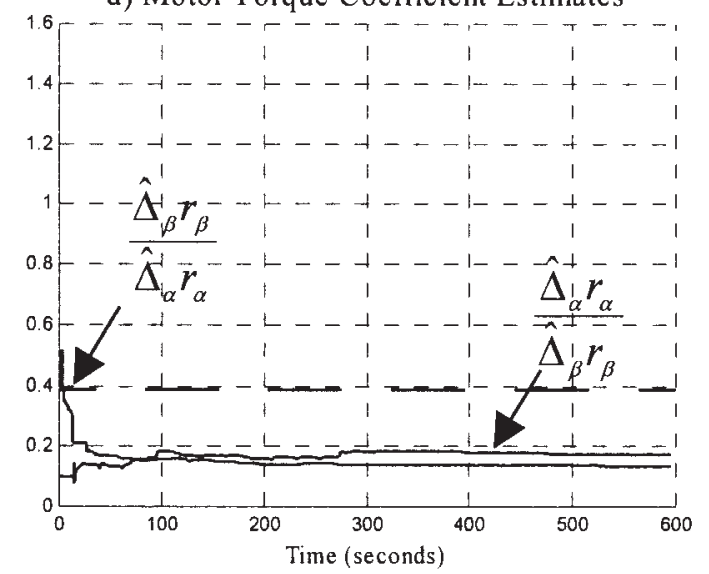

Fig. 14 Experimental parameter estimates with user-input 3

Fig. 9a). Similarly, Figs $11 \mathrm{~b}$ and c illustrate the comparison for the user-inputs given by Figs $9 \mathrm{~b}$ and c respectively. Computed-torque control was performed with user-inputs similar to those given in Fig. 9. The torque command could not be reproduced accurately owing to the input being applied manually. For clarity, the computed-torque controller error is shown offset by $0.005 \mathrm{rad}$. It can be seen that in the cases of Figs $11 \mathrm{~b}$ and $\mathrm{c}$ there is not a significant difference in the performance of the composite adaptive controller compared to that of the computed-torque method.

The parameter estimates corresponding to the results of the user-input of Figs 9a, b and $\mathrm{c}$ are given in Figs 12,13 and 14 respectively. Unlike the simulation, the parameter errors converge on different values to those calculated in equation (48) and shown by a dotted line in each figure. In the case of the low-magnitude userinput (Fig. 12) the parameters are slow to reach a constant estimate. However, for the two larger magnitude user-input commands, Figs 13 and 14, the parameter errors are shown to reach steady state more rapidly.

The simulation and experimental results are different. It has not been the aim of this work to fully simulate the whole system accurately, including the digital interface, but simply to use the simulation to develop the control strategy, which is then implemented practically. For example, the friction cannot be modelled precisely and the simulation model does not include flexibility in the joint mechanism and assumes perfect measurement of motor torque and speed. Despite these issues the composite adaptive controller is shown to work well and is robust against both modelling and measurement errors.

\section{CONCLUSIONS}

Both simulation and experimental results have shown that a composite adaptive control scheme can be used to track a desired path. It has been shown that the dependence of the desired velocity on the user-input force and error in the model has a detrimental effect on the convergence of the tracking and parameter errors to zero. The composite adaptive control method has been shown to give a performance equivalent to the computed-torque algorithm [5] under certain conditions on the user-input torque command. It has been shown experimentally that if the desired velocity and user-input are not sufficiently 
independent of each other, the composite adaptive controller does not perform as well as the computed-torque algorithm. However, in both the simulation and experimental results, the tracking and parameter errors remain bounded under these conditions. The dual-worm mechanism is still able to cancel backlash using the composite adaptive control scheme.

Unlike the composite adaptive controller, the ability of the computed-torque control algorithm to track a path relies on fixed estimates of the joint parameters. Therefore, degradation of the computed-torque controller's performance due to wear of the gears or changes in load can be expected. This would require periodic re-tuning of the algorithm in order to ensure satisfactory performance, which can be a time consuming process. The composite adaptive control scheme has been shown to be robust in the face of large modelling and measurement errors, and as such can be used to compensate for changes in system parameters. Due to the nature of the proposed application, it may not be prudent to allow the controller to adjust parameters during operation. The composite adaptive controller should be used to train the manipulator off-line as part of a calibration procedure before it is used. Care must be taken to ensure that the user-input command consistently saturates the desired velocity function during this training period.

\section{REFERENCES}

1 Davies, B. L. A discussion of safety issues for medical robots. In Computer-Integrated Surgery (Eds R. H. Taylor, S. Lavallée, G. C. Burdea and R. S. Mösges), 1996, pp. 287-296 (MIT Press, Cambridge, Massachusetts).
2 Troccaz, J. and Delnondedieu, Y. Semi-active guiding systems in surgery. A two degree-of-freedom prototype of the passive arm with dynamic constraints (PADyC). Mechatronics, 1996, 6(4), 399-421.

3 Moore, C. A., Peshkin, M. A. and Colgate, J. E. A three revolute Cobot using CVTs in parallel. In Proceedings of the ASME International Mechanical Engineering Congress and Exposition (IMECE), Nashville, Tennessee, 14-19 November 1999.

4 Harris, S. J., Lin, W. J., Fan, K. L., Hibberd, R. D., Cobb, J., Middleton, R. and Davies, B. L. Experiences with robotic systems for knee surgery. Lecture Notes in Computer Sci., 1997, 1205, 757-766.

5 Reedman, A. V. C. and Bouazza-Marouf, K. Control of an actively constrained robotic joint for passive deployment applications. Proc. Instn Mech. Engrs, Part K, Journal of Multi-body Dynamics, 2001, 215( K4), 187-197.

6 Patton, W. J. Mechanical Power Transmission, 1980 (Prentice-Hall, Englewood Cliffs, New Jersey).

7 Bo, L. C. and Pavelescu, D. The friction-speed relationship and its influence on the critical velocity of stick-slip motion. Wear, 1982, 82, 277-289.

8 Craig, J. J., Hsu, P. and Sastry, S. S. Adaptive control of mechanical manipulators. Int. J. Robotics Res., 1987, 6(2), 16-28

9 Slotine, J. J. and Li, W. Adaptive robot control: a new perspective. In Proceedings of the IEEE 28th International Conference on Decision and Control, Los Angeles, California, December 1987, pp. 192-198.

$10 \mathbf{~ L i}, \mathbf{W}$. and Slotine, J. J. Parameter estimation strategies for robotic applications. In ASME Winter Annual Meeting-Dynamic Systems and Control Division, 1987, pp. 213-218.

11 Marino, R. and Tomei, P. Nonlinear Control Design: Geometric, Adaptive and Robust, 1995 (Prentice-Hall, Englewood Cliffs, New Jersey).

12 Yager, T. The QNX operating system. Byte, August 1990, 15(8), 281-283. 